\title{
Detecting Financial Collapse and Ballooning Sovereign Risk*
}

\author{
Peter C. B. Phillips \\ Yale University, University of Auckland, \\ University of Southampton $\&$ Singapore Management University \\ Shuping Shi \\ Macquarie University
}

February 25, 2019

\begin{abstract}
This paper proposes a new model for capturing discontinuities in the underlying financial environment that can lead to abrupt falls, but not necessarily sustained monotonic falls, in asset prices. This notion of price dynamics is consistent with existing understanding of market crashes, which allows for a mix of market responses that are not universally negative. The model may be interpreted as a martingale composed with a randomized drift process that is designed to capture various asymmetric drivers of market sentiment. In particular, the model is capable of generating realistic patterns of price meltdowns and bond yield inflations that constitute major market reversals while not necessarily being always monotonic in form. The recursive and moving window methods developed in Phillips, Shi and Yu (2015a,b, PSY), which were designed to detect exuberance in financial and economic data, are shown to have detective capacity for such meltdowns and expansions. This characteristic of the PSY tests has been noted in earlier empirical studies by the present authors and other researchers but no analytic reasoning has yet been given to explain why methods intended to capture the expansionary phase of a bubble may also detect abrupt and broadly sustained collapses. The model and asymptotic theory developed in the present paper together explain this property of the PSY procedures. The methods are applied to analyze S\&P500 stock prices and sovereign risk in European Union countries over 2001-2016 using government bond yields and credit default swap premia. A pseudo real-time empirical analysis of these data shows the effectiveness of the monitoring strategy in capturing key events and turning points in market risk assessment.
\end{abstract}

Keywords: Collapse, Crash, Exuberance, Recursive test, Rolling test, Sovereign risk JEL classification: $\mathrm{C} 23$

${ }^{*}$ Phillips acknowledges research support from the Kelly Fund, University of Auckland. Shi acknowledges the research support from the Australian Research Council [project number DP150101716]. Peter C.B. Phillips email: peter.phillips@yale.edu. Shuping Shi, email: shuping.shi@mq.edu.au. 


\section{Introduction}

Crashes are often defined in terms of an abrupt discontinuity in the relationship between the underlying financial environment and stock prices that produces an unusually large negative movement in asset prices (Gennotte and Leland (1990); Barlevy and Veronesi (2003); Hong and Stein (2003)). Abrupt movements in market prices of this type may well produce a major initial unidirectional change but are seldom monotonic for a sustained period, a phenomenon that complicates modeling and econometric inference. In this regard, market collapses share a common feature with the expansionary phase of most bubbles.

The occurrence of financial market crashes may be due to significant news events or reported changes in fundamentals. The sudden and disruptive re-pricing of Euro area sovereign credit risk in 2008-2012 is a vivid example. Starting from Ireland in late September 2008, Euro area governments announced a set of rescue packages. These measures commonly took the form of capital injections and guarantees for financial sector liabilities and purchases of illiquid assets from financial institutions, all intended to increase confidence in their banking systems. According to the International Monetary Fund, as of April 15, 2009, the total support for the financial sector in Ireland reached a level of 2.63 times its 2008 GDP. ${ }^{1}$ While the effect of these fiscal interventions on national economies is more difficult to measure, it is clear that the interventions led to significant deteriorations in budget positions and a ballooning of government debt. The average upfront financing need cited in the IMF report for the Euro countries in April 2009 was around five percent of 2008 GDP, and upfront Government financing in the UK exceeded 20\%. The resulting negative shock to the public sector caused tremendous falls in government bond prices, with bond yields and credit default swaps soaring to record highs over the immediate years following the crisis, particularly in Southern European countries.

On the other hand, many of the dramatic crashes in the US stock market -most notably the 1929 and 1987 crashes -are documented to occur without any particular significant news events or fundamental changes (Cutler et al. (1989) and Gennotte and Leland (1990)). There is a vast literature providing alternative explanations for crash discontinuities in stock prices. For example, Gennotte and Leland (1990) show that in a market where uninformed investors are unable to distinguish hedging activity from information-based trades, large numbers of such investors may revise downward their expectations when there are what appear to be infinitesimal shifts in information or other small shocks that lead to lower prices. The pessimistic view of the market limits their willingness to absorb the extra supply and causes a magnified price response. In a behavioral model with heterogeneous traders that take exuberant, cautious, and neutral views of the same fundamentals, Phillips (2016) shows that price solution paths may alternate between exuberance and collapse, depending on the relative proportions of such traders. Small shifts alone in these proportions can have a major impact on market direction.

Barlevy and Veronesi (2003) show that, even in the absence of hedging strategies such as stoploss strategies and portfolio insurance, uninformed traders can in some circumstances precipitate a price crash. In related work, Romer (1993) and Hong and Stein (2003) consider a market environment where investors possess diverse useful information about fundamentals. The models

\footnotetext{
${ }^{1}$ http://www.imf .org/external/np/fad/2009/042609.htm.
} 
proposed by Romer (1993) and Hong and Stein (2003) share a common spirit that the trading process causes endogenous revelation of private information, which in turn can lead to large price changes based on only small observable news events when many investors are affected. In a similar manner, Cont and Wagalath $(2013,2016)$ note that the sudden deleveraging of large financial portfolios (or fire sales) can be self-reinforcing, leading to a downward spiral in asset prices.

Taken together, all of this research points to multifarious potential sources of market reversals, often involving only small shifts in behavior, perception, or the availability of new information. Against this complex background, the challenge of modeling market collapse is considerable, particularly when the factors driving change are unobservable, often behavioral, and commonly the outcome of small cumulative shifts in participant thinking and incoming news on market fundamentals.

The approach adopted in the present paper involves a new reduced form dynamic for asset prices during periods of disruptive market crashes where the multiplicity of unobservable drivers is captured through the arrival of asymmetric shocks that augment the usual martingale model for asset prices. The data generating process (DGP) is consistent with the crash definition of Gennotte and Leland (1990). In particular, the collapse process is assumed to be driven by the arrival of new shocks to the market that are independent of past data and that reflect broadly negative market sentiment appearing in the market. We show that this DGP is capable of generating both exogenous (or news-led) crises as well as endogenous crises. The random-drift augmented martingale is of some independent interest and generalizes the usual martingale and martingale plus drift mechanisms that are commonly used in other work.

This new model also differs from the (stationary) mildly integrated process model used in earlier work by Phillips and Shi (2018; 2017, PS) and Harvey et al. (2016), where the historical sample path of the data series matters but is attentuated in a way that reflects reduced market expectations. Although both collapse processes can produce realistic patterns of market behavior during crisis episodes where market collapses occur, the new model is especially well-suited to capturing crash behavior where there are abrupt falls in asset prices. Correspondingly, the asymptotic properties and implications of the two processes are very different.

One particularly useful aspect of the new model is that it explains an interesting empirical feature of the bubble detection procedure of Phillips, Shi and Yu (2015a; 2015b, PSY). The recursive rolling detection procedure of PSY was designed to detect speculative bubbles during the expansion phase and to consistently estimate origination and termination dates of such bubbles. We show both asymptotically and in finite samples that this procedure also has detective power against the new data generating process for a market crash and that it can consistently estimate the dates of the crash. The crash detection capability of the PSY procedure has been noted in several recent empirical articles by the authors (PSY (2015a); PS (2018); Shi (2017); Deng et al. (2017)) and by many other researchers considering stock prices, exchange rates and other financial time series where abrupt crashes and sustained collapses have occurred. See, for example, Yiu and Jin (2013), Fantazzini (2016), Hu et al. (2016); Hu and Oxley (2018), Wegener et al. (2017). This capability of PSY is further illustrated in the present paper with an application of the procedure to the S\&P500 stock market over the period of January 2005 to March 2009.

Crashes in asset prices are mirrored in bond yield inflation. The PSY procedure is, therefore, 
well-suited as a real-time monitoring device for ballooning sovereign risks. Sovereign risk is acknowledged to pose a serious threat to the stability of the global economic and financial system. The past decade has been a particularly unsettling period for the public sector in Europe. The bailout packages that were implemented during and following the financial crisis opened up considerable new financial linkages between the public and banking sectors, raising sovereign risk to historical highs. Especially in Greece, rising sovereign risk led to ballooning government bond yield and credit default swap premiums; and the unprecedented level of public debt announced in December 2009 triggered a prolonged period of panic and sustained economic recession in the country. This unusual period of economic and financial upheaval is difficult to model with traditional methods and is a convenient vehicle for demonstrating the usefulness of the model and inferential methodology introduced in the present paper.

Using 10-year government bond yields and five-year credit default swap premia as proxies for sovereign risk, we conduct a pseudo real-time monitoring exercise of sovereign risk in Portugal, Greece, Ireland, Italy and Spain (PIIGS) over the period from January 2001 to September 2016. The PSY procedure identifies both the 2007-2008 and the 2010-2012 crisis episodes in all PIIGS countries. The warning signal is switched on by the test as early as July 2007 in the CDS market (September 2007 in the bond market) and again for all PIIGS countries soon after the Greek announcement of a 300 billion national debt (exceeding the entire Greek economy) led to a downgrade by rating agencies of Greek debt to junk status with wide repercussions for other European economies, foreshadowing the European debt crisis.

The paper is organized as follows. Section 2 introduces the new econometric model for capturing price crashes and yield inflation, along with various limit properties associated with the model that facilitate testing. We calibrate the model to the S\&P 500 stock market prices during the subprime mortgage crisis period and to Greek 10-year government bond yields during the European debt crisis period. Section 3 describes the recursive rolling procedure of PSY and investigates the asymptotic and finite sample performance of the procedure under the proposed data generating process for price crashes. The crash detection capability of the PSY test is demonstrated in Section 4 with an application to the S\&P500 stock market. Section 5 conducts a pseudo real-time monitoring of sovereign risk in the PIIGS countries, with both the 10-year government bond yield and the 5-year credit default swaps. Section 6 concludes. Proofs and other technical material are in the Appendix.

\section{An Econometric Model for Price Collapse and Yield Inflation}

In the spirit of Gennotte and Leland (1990) and Barlevy and Veronesi (2003), we can think of a price crash as a discontinuity in the pricing function originating from a change in one of its continuous arguments, producing what might end up being a very large response to a small change in fundamentals or perceptions of fundamentals. Barlevy and Veronesi provide a mechanism for such changes in terms of a disjointed locally non-monotonic demand curve (locally upward sloping and cutting off at some price level) for an asset associated with uninformed traders making noisy decisions in the face of risk.

In a related way, but in a reduced form framework, the econometric model we employ mod- 
ifies the usual efficient market price mechanism by introducing asymmetric shock effects that supplement fundamentals. In particular, in our model the logarithmic price change or asset return $\left(\log P_{t}-\log P_{t-1}\right)$ is driven by the superposition of martingale differences and a random sequence $\left(-L_{t}\right)$ that is temporarily and locally asymmetric in the negative direction, embodying trader negative sentiment on the direction of fundamentals. We use a prototypical set up to demonstrate the capability of this model to capture forces that can precipitate a market price crash. The model is specifically useful in analyzing the asymptotic behavior of econometric tests for collapse during such episodes.

The model is constructed to have the following specific form during crash episodes

$$
\log P_{t}-\log P_{t-1}=-L_{t}+\varepsilon_{t}
$$

where the $\varepsilon_{t}$ are martingale difference sequence (mds) innovations with variance $\sigma^{2}$, the random sequence $L_{t}$ follows a scaled uniform distribution, and $\varepsilon_{t}$ and $L_{t}$ are assumed independent. We assume that $L_{t}$ takes the form

$$
L_{t}=L b_{t}, \text { with } b_{t} \sim_{i i d} U[-\epsilon, 1] \text { and } \epsilon<1
$$

where $L$ is a positive scale quantity measuring shock intensity and $b_{t}$ is uniform on an interval ranging from a (usually small) negative value $-\epsilon$ to unity. The average return during market crashes is therefore negative and is given by $-L(1-\epsilon) / 2$. We refer to this collapse process (1) as the $L$-process. The support of $b_{t}$ is asymmetric around zero, a design that produces predominantly negative shocks in the measure $-L_{t}$ in (1). These negative shocks are mixed with some positive shocks in $-L_{t}$ that occur with smaller probability controlled by the magnitude of the parameter $\epsilon$. This specification, as demonstrated below, leads to realistic collapse trajectories that are by no means necessarily monotonic. Abrupt major crashes are captured with (1) by allowing the scale parameter $L$ to diverge (i.e. $L \rightarrow \infty$ ). We refer to $(1)$ under this setting as the $L^{\infty}$-process.

The $L$-process in (2) induces a form of randomized drift in the time series with direction determined by the signed asymmetry and intensity determined by $L$. This random drift component can be further decomposed into two elements such that

$$
L_{t}=\underbrace{E\left(L_{t}\right)}_{\text {deterministic drift }}+\underbrace{\left[L_{t}-E\left(L_{t}\right)\right]}_{\text {noise }} .
$$

While the deterministic drift component controls the impact of $L_{t}$ on the mean of asset returns, the noise component adds to the volatility of returns. This is consistent with the empirical fact that negative mean and larger volatility are typically observed for returns during crisis periods (Forbes and Rigobon, 2002; Dungey et al., 2005). In consequence, during crisis episodes the log price process follows a mean-shifted martingale process in which returns satisfy

$$
\log P_{t}-\log P_{t-1}=-E\left(L_{t}\right)+\eta_{t},
$$

where $\eta_{t}=\varepsilon_{t}+\left[L_{t}-E\left(L_{t}\right)\right]$ is an mds with variance $\sigma^{2}+(1+\epsilon)^{2} / 12$.

Many variants of the prototypical specification (2) are possible. In particular, the support of $b_{t}$ controls the general form of the data trajectories produced by the model. For instance, the 
paths become progressively closer to those of a martingale with (monotonic) linear drift as the support narrows to a single point, showing that simpler variants of martingale models with some (potentially noisy) deterministic drift are subsumed within the formulation as $\epsilon \rightarrow-1$. Higher order randomized drifts are also included in this framework by simple extension of the definition $(2) .^{2}$

Moreover, the specification (1) may be such that the $L$-process switches on or off depending on the financial environment and trader sentiment. This switching behavior is controlled by the scale parameter $L$, which takes the value zero during normal periods or a positive number when the market sustains declines or collapses.

\subsection{Stock Market Crashes}

Denote the date of market collapse by $T_{c}=\left\lfloor T r_{c}\right\rfloor$ for some sample fraction $r_{c}>0$ where the floor function $\lfloor\cdot\rfloor$ gives the integer part of its argument. The logarithmic asset price in a two-regime framework then follows the generating mechanism

$$
\log P_{t}=\left\{\begin{array}{ll}
c T^{-\eta}+\log P_{t-1}+\varepsilon_{t} & \text { if } t \in N \equiv\left[1, T_{c}\right] \\
-L_{t}+\log P_{t-1}+\varepsilon_{t} & \text { if } t \in C \equiv\left(T_{c}, T\right]
\end{array} .\right.
$$

In the normal regime (denoted $N$ ), the scale parameter $L$ is zero and the log price is assumed to follow a martingale with an asymptotically negligible drift (i.e. $c T^{-\eta}$ with constant $c$ and $\eta>1 / 2$ ), designed to capture a slow drift in the market over time, as used in PSY and PS. When the market collapses (denoted as regime $C$ ), the log price switches to the $L$-process dynamic (1).

The representation (4) shows that the model (5) may be interpreted in terms of a structural break model for asset returns. However, as is well known, small mean shift deviations in returns can be very difficult to detect because return volatility, which is typically amplified during crisis episodes, tends to mask small shifts in mean. On the other hand, working with the price series, or price series normalized by fundamentals, enables comparison between bubble and crash episodes in the data using existing test procedures such as those in PSY. The formulation (1) is then particularly useful in such comparisons.

We calibrate DGP (5) to the log S\&P 500 price index over the famous subprime mortgage crisis period. Data is obtained from DataStream International. The US stock market reached historical highs in mid-2007 and started to tumble from the second half of 2007. We therefore use the period 2005M01-2007M07 for the normal $(N)$ regime and the period 2007M08-2009M02 for the crisis $(C)$ regime. This simulation uses $i i d\left(0, \sigma^{2}\right)$ innovations $\varepsilon_{t}$ and the calibrated parameter settings are $X_{0}=7.08, c T^{-\eta}=0.009, \sigma=0.024, L=0.17, \epsilon=0.63, T_{c}=31$ and $T=50$. Figure 1b plots a typical realization of (5) and Figure 1a plots the log S\&P 500 price index for comparison. As evident from these graphs, the new DGP is capable of generating empirically realistic patterns of serious market downturns.

\footnotetext{
${ }^{2}$ For example, the $L$-process produces a randomized quadratic drift in the time series when $L_{t}=L_{t}^{*} b_{t}$, with $L_{t}^{*}=L_{a_{1}}+L_{a_{2}} t$ and constant coefficients $\left(L_{a_{1}}, L_{a_{2}}\right)$, which specializes to a non-random quadratic drift when $\epsilon \rightarrow-1$. This formulation can be further extended by employing coefficients $L_{a_{1}, t}, L_{a_{1}, t}$ which are individually randomized in the same manner as $L_{t}$ but with their own uniformly and asymmetrically distributed scale factors.
} 
Figure 1: The logarithmic S\&P 500 price index running from January 2005 to March 2009 and a typical realization of the data generating process (5).

(a) $\log \mathrm{S} \& \mathrm{P} 500$ price index

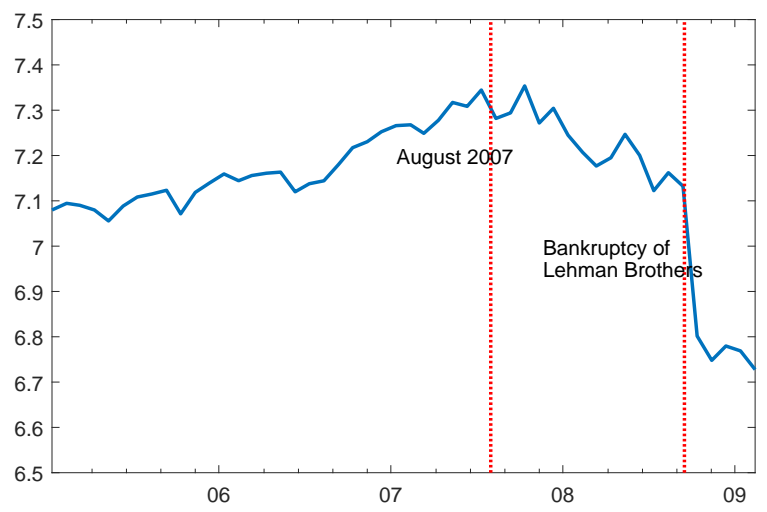

(b) Simulated $L$-process sample path

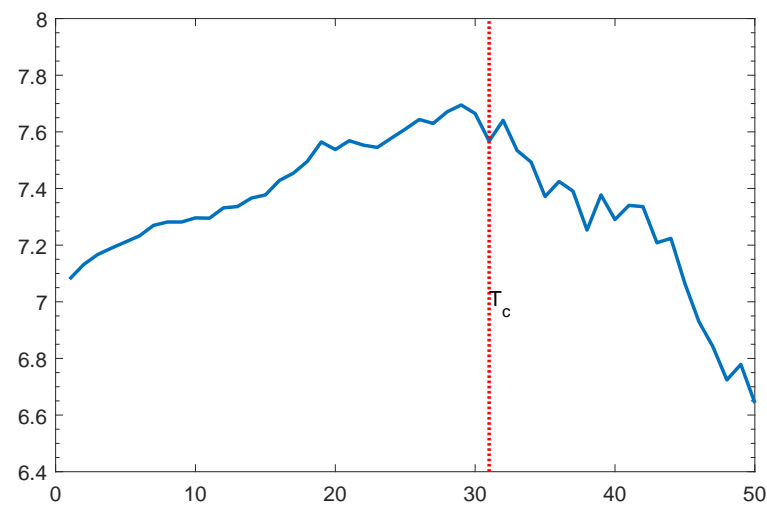

The model is readily modified to incorporate fundamentals. In particular, suppose the logarithmic dividend $\left(D_{t}\right)$ process follows a random walk with drift so that

$$
\log D_{t}-\log D_{t-1}=\alpha+v_{t} .
$$

where $\alpha$ is a constant and the $v_{t}$ are mds innovations. Under the price process (1), the logarithmic price-dividend ratio also follows a random drift martingale process of the form

$$
\log P_{t} / D_{t}=-L_{t}^{*}+\log P_{t-1} / D_{t-1}+\varepsilon_{t}^{*},
$$

where the $L$-process is $L_{t}^{*}=L b_{t}^{*}$ with $b_{t}^{*} \sim_{i i d} U[-\epsilon+\alpha / L, 1+\alpha / L]$ and $\varepsilon_{t}^{*}=\varepsilon_{t}-v_{t}$.

\subsection{Ballooning Sovereign Risks}

Suppose $P_{t}$ is the price of a $\tau$-period discount bond and $y_{t}$ is the continuously compounded zero-coupon nominal yield to maturity. The relationship between the bond price and its yield is $P_{t}=\exp \left(-\tau y_{t}\right)$. This implies an inverse linear relationship between the bond yield and logarithmic price such that $y_{t}=-\frac{1}{\tau} \log P_{t}$. Assuming a collapse process (1) for bond prices, $y_{t}$ follows the dynamic

$$
y_{t}-y_{t-1}=\frac{1}{\tau} L_{t}+v_{t} \text { with } v_{t} \sim_{i i d} N\left(0, \sigma^{2} / \tau\right)
$$

Unlike the process for bond prices, the $L$-component of the bond yield (i.e. $\frac{1}{\tau} L_{t}$ ) is positive which produces an inflationary overall pattern to bond yields during periods of credit crunch.

Next, suppose that the asset of interest is a discount bond and its price follows DGP (5). The bond yield $y_{t}$ then satisfies the two-regime mechanism

$$
y_{t}= \begin{cases}-c \tau T^{-\eta}+y_{t-1}+v_{t} & \text { if } t \in N \equiv\left[1, T_{c}\right] \\ y_{t-1}+\frac{1}{\tau} L_{t}+v_{t} & \text { if } t \in C \equiv\left(T_{c}, T\right]\end{cases}
$$


which generates bond yield inflation via the scaled and positively signed $L$-process $(1 / \tau) L_{t}$. It follows that appropriate signing of the $L$-process generating mechanism allows for both price collapse episodes and ballooning yield data trajectories.

Figure 2: Greek 10-year government bond yields from January 2006 to February 2012 and a typical realization of the DGP (9).

(a) Greek 10-year government bond yields

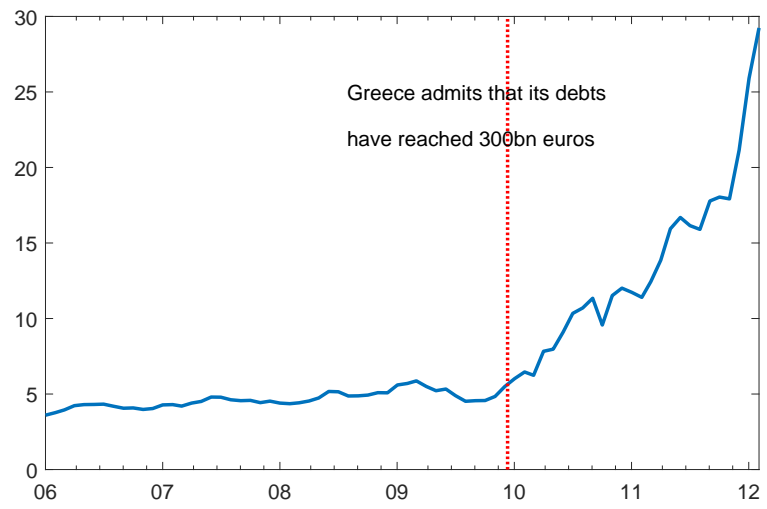

(b) Simulated $L$-process sample path

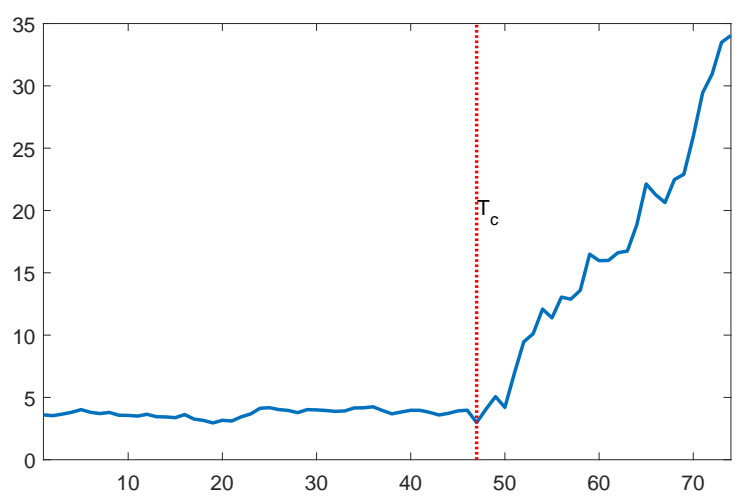

To visualize the realism of the data generating process, we calibrate (9) to the Greek 10-year government bond yield from January 2006 to February 2012. Data are obtained from DataStream. The sample period covers the European debt crisis period. The announcement of a 300 billion euro debt by the Greek government in December 2009 triggered a round of aggressive expansion in bond yields. We divided the sample into two periods: 2006M01-2009M11 (regime $N$ ) and 2009M122012M02 (regime $C$ ) with calibrated parameter settings $y_{0}=3.6, \tau=120,-c \tau T^{-\eta}=0.03$, $\sigma=2.16, L=391.79, \epsilon=0.45, T_{c}=47$ and $T=74$.

A simulated sample path and the Greek 10-year government bond yield are plotted in Figure 2 b. It is evident from these graphs that, when the $L$-process is appropriately signed as in (9), the mechanism can equally well generate patterns of noisy upward expansion.

\subsection{Model Behavior Under Different Collapse Regimes}

This section examines some key properties of log prices under the DGP (5) when a market crash occurs after a period of normal martingale dynamics. To aid the development we make the following assumptions.

A1: $\varepsilon_{t}$ is iid with zero mean and $\mathbb{E} \varepsilon_{t}^{2}=\sigma^{2}<\infty$.

A2: $\varepsilon_{t}$ is an mds satisfying:

(i) $\varepsilon_{t}$ is strongly uniformly integrable with a dominating random variable $\eta$ that satisifies $\mathbb{E}\left(\eta^{2} \ln ^{+}|\eta|\right)<\infty$

(ii) $T^{-1} \sum_{t=1}^{T} \mathbb{E}\left(\varepsilon_{t}^{2} \mid \mathcal{F}_{t-1}\right) \rightarrow_{\text {a.s. }} \sigma^{2}$, where $\mathcal{F}_{t}=\sigma\left\{\varepsilon_{t}, \varepsilon_{t-1} \ldots\right\}$ is the natural filtration. 
A1 assumes homoskedastic independent innovations and A2 allows for conditional heteroskedasticity. Under $\mathbf{A} \mathbf{2}\left\{\varepsilon_{t}\right\}$ is serially uncorrelated and unconditionally homoskedastic if $\mathbb{E}\left(\varepsilon_{t}^{2}\right)=\sigma^{2}$ for all $t$ (and hence covariance stationary in that case), but potentially conditionally heteroskedastic. A2 allows, among other possibilities, stable ARCH or GARCH errors. ${ }^{3}$ From Phillips and Solo (1992), under either A1 or A2 together with the conditions on $b_{j}$ we have the following invariance principles

$$
T^{-1 / 2} \sum_{t=1}^{\lfloor T \cdot\rfloor} \varepsilon_{t} \Rightarrow B(\cdot)=: \sigma W(\cdot), T^{-1 / 2} \sum_{t=1}^{\lfloor T \cdot\rfloor} \xi_{t} \Rightarrow B_{\xi}(\cdot)=: \sigma_{\xi} W_{\xi}(\cdot),
$$

where $W$ and $W_{\xi}$ are independent standard Brownian motions and the floor function $\lfloor\cdot\rfloor$ returns the integer part of its argument.

As shown in Lemma A.3 of the Appendix, when the collapse process follows $L_{t}$ as in (2), asset price dynamics take the following different forms in normal and crash regimes:

(i) For $t \in N, \log P_{t=\lfloor T r\rfloor}=\sum_{s=1}^{\lfloor T r\rfloor} \varepsilon_{s}\left\{1+o_{p}(1)\right\} \sim_{a} T^{1 / 2} B(r)$.

(ii) For $t \in C, \log P_{t=\lfloor T r\rfloor}=-\sum_{j=T_{c}+1}^{\lfloor T r\rfloor} L_{j}\left\{1+o_{p}(1)\right\} \sim_{a}-T L \frac{1}{2}\left(r-r_{c}\right)(1-\epsilon)$.

Martingale behavior in the normal regime is standard. But when observations lie in the crisis regime, the composition of asset prices involves several components

$$
\log P_{t}=\underbrace{\log P_{T_{c}}}_{\text {initial condition }}-\underbrace{\sum_{j=T_{c}+1}^{t} L_{j}}_{\text {L-component }}+\underbrace{\sum_{j=T_{c}+1}^{t} \varepsilon_{j}}_{\text {stochastic trend }},
$$

with each component having its own driver elements. The $L$-process component in (11) dominates both the initial condition and the stochastic trend, diverging to negative infinity at rate $O_{p}(T)$ for the $L$-process with fixed $L$ and at rate $O_{p}(T L)$ when $L \rightarrow \infty$. The bond yield, by definition, will diverge to positive infinity at the same rate. The model therefore accommodates scenarios of collapse or exuberance with rates and direction controlled by the behavior of $L$. As is apparent from the form of (11), the crash process includes simple drift mechanisms as a special case when the $L$-process is nonstochastic, producing a linear drift ${ }^{4}$ as a special case, for instance, when $\epsilon \rightarrow-1$.

This model differs substantially from the bubble collapse process used in PS (2018a,b). While the L-process in (1) generates collapse patterns that are independent of the underlying asset

\footnotetext{
${ }^{3}$ Note that A2 does not allow for unconditional heteroskedasticity. Harvey et al. (2016) show that the empirical size of the test could potentially be affected by the presence of unconditional heteroskedasticity such as structural changes in the unconditional variance. Several approaches have been proposed to address this issue (Harvey et al., 2016, ming, 2018). In particular, the wild bootstrapping procedure of Harvey et al. (2016) is found to have satisfactory asymptotic and finite sample performance.

${ }^{4}$ Here, $\sum_{j=T_{c}+1}^{t} L_{j} \rightarrow L\left(t-T_{c}-1\right)$ as $\epsilon \rightarrow-1$. As indicated earlier, higher order polynomial drifts are produced in a similar fashion upon suitable definition of the deterministic component of $L_{j}$.
} 
price dynamics, the PS process produces path-dependent trajectories. In PS, bubble implosion is modeled by a (stationary) mildly integrated process (Phillips and Magdalinos, 2007, 2009). Specifically,

$$
\log P_{t}=\left\{\begin{array}{ll}
d T^{-\eta}+\log P_{t-1}+\varepsilon_{t} & \text { if } t \in N \equiv\left[1, T_{c}\right] \\
\gamma_{T} \log P_{t-1}+\varepsilon_{t}, & \text { if } t \in C \equiv\left(T_{c}, T\right]
\end{array} .\right.
$$

where $\gamma_{T}=1-c T^{-\beta}$ with localizing (to unity) coefficient $c>0$ and $\beta \in[0,1) .{ }^{5}$ Under this model, the dynamics of $\log$ prices in regime $C$ can be rewritten as

$$
\log P_{t}=\underbrace{\gamma_{T}^{t-T_{c}} \log P_{T_{c}}}_{\text {initial condition }}+\underbrace{\sum_{j=0}^{t-T_{c}-1} \gamma_{T}^{j} \varepsilon_{t-j}}_{\text {stochastic shocks }} .
$$

Given that $\gamma_{T}<1$, the impact of the random shocks $\varepsilon_{t}$ diminishes as time elapses; and the speed at which the diminution occurs increases as the parameter $\beta$ becomes smaller.

Under the generating mechanism (12) and with $\varepsilon_{t}$ satisfying either Assumption A1 or A2, asset price dynamics take the following form in the crash regime for $t \in C$,

$$
\log P_{t=\lfloor T r\rfloor}=\sum_{j=0}^{t-T_{c}-1} \gamma_{T}^{j} \varepsilon_{t-j}\left\{1+o_{p}(1)\right\} \sim_{a} T^{\beta / 2} X_{c}
$$

with $X_{c} \equiv N\left(0, \sigma^{2} / 2 c\right)$. The limit theory follows directly from Phillips and Magdalinos $(2007)^{6}$ and in this case the initial condition is dominated asymptotically by the reverting component. Thus, standardized log prices converge to a normal variate with mean zero at the rate $O_{p}\left(T^{\beta / 2}\right)$ and with variance dependent on the localizing coefficient $c$ in the mildly integrated component of (12). Evidently, the order of magnitude of $\log P_{t}$ in regime $\mathrm{C}$ is much higher under DGP (5) than under DGP (12), with the $L$-process placing direct downward pressure on prices. This difference can have a considerable impact on price trajectories under collapse regimes, particularly when the intensity parameter $L$ is large, which enables more realistic representation of prices during abrupt crises with dramatic collapses in prices. The difference also has a major effect on the asymptotic behavior of test and detection procedures, as will be discussed below.

\section{The PSY Procedure for Detecting Crashes}

The PSY procedure has been applied to a wide variety of financial and real estate markets for bubble monitoring [see, for example, Gutierrez (2012); Fantazzini (2016); Etienne et al. (2013); Pavlidis et al. (2016); Shi et al. (2016)]. The methodology and warning alert system has also received attention from policy makers ${ }^{7}$ and the financial press (Meyer, 2013). The method has

\footnotetext{
${ }^{5}$ Harvey et al. (2016) set the localizing exponent coefficient $\beta$ to be zero, so that the process in regime $C$ is a fixed stationary process rather than mildly integrated.

${ }^{6}$ See Phillips and Shi $(2018 \mathrm{a}, \mathrm{b})$ for related work on bubble implosion asymptotics.

${ }^{7}$ For example, the quarterly exuberance indicators published by the Federal Reserve Bank of Dallas for 23 international housing markets (http://www.dallasfed.org/institute/houseprice/) are based on the PSY procedure.
} 
been successfully employed both as a crisis dating device and as an early warning alert system for market exuberance.

The rolling-recursive procedure of PSY is based on a right-tailed unit root test (Phillips et al., 2014). The null hypothesis captures normal market behavior and states that the logarithmic asset price follows a martingale process with a mild drift function such that

$$
\log P_{t}=g_{T}+\log P_{t-1}+\varepsilon_{t}
$$

where $g_{T}=k T^{-\gamma}$ with constant $k$ and $\gamma>1 / 2$. The drift term captures any mild drift that may be present in prices but which is of smaller order than the martingale component and is therefore asymptotically negligible. The regression model used for estimation and inference includes an intercept but no time trend and has the following form ${ }^{8}$

$$
\log P_{t}=\mu+\rho \log P_{t-1}+\varepsilon_{t},
$$

where $\varepsilon_{t} \stackrel{m d s}{\sim}\left(0, \sigma^{2}\right)$ or, more generally, the conditions detailed in Assumption A2. Suppose the regression runs from $T_{1} \equiv\left\lfloor r_{1} T\right\rfloor$ to $T_{2} \equiv\left\lfloor r_{2} T\right\rfloor$. The corresponding subsample unit root (t-ratio) test statistic is denoted by $D F_{r_{1}}^{r_{2}}$ and defined as

$$
D F_{r_{1}}^{r_{2}}=\frac{\sum_{j=T_{1}}^{T_{2}} \tilde{X}_{j-1}\left(\tilde{X}_{j}-\tilde{X}_{j-1}\right)}{\left[\hat{\sigma}_{r_{1} r_{2}}^{2} \sum_{j=T_{1}}^{T_{2}} \tilde{X}_{j-1}^{2}\right]^{1 / 2}},
$$

where $\tilde{X}_{j-1}$ is the demeaned log price and $\hat{\sigma}_{r_{1}, r_{2}}^{2}$ is the least squares (OLS) estimator of the error variance of the regression over $\left[T_{1}, T_{2}\right]$. Importantly, the sign of the DF statistic is determined by the numerator.

For each observation of interest $\lfloor r T\rfloor$, corresponding to some sample fraction $r$, we construct a backward expanding subsample sequence with the end point of the regression fixed on $\lfloor r T\rfloor$ (i.e., we set $r_{2}=r$ ) and allow the starting point to vary within its feasible range such that $r_{1} \in\left[0, r-r_{0}\right]$, where $r_{0}$ is the minimum window size required to initiate the regression. The test statistic for $\lfloor r T\rfloor$ is denoted by $B S D F_{r}\left(r_{0}\right)$ and defined as

$$
B S D F_{r}\left(r_{0}\right)=\sup _{r_{1} \in\left[0, r-r_{0}\right], r_{2}=r}\left\{D F_{r_{1}}^{r_{2}}\right\} .
$$

Under the null hypothesis, the BSDF statistic has a limit distribution determined by the limiting form of the sup variate, viz.,

$$
F_{r}\left(r_{0}\right):=\sup _{r_{1} \in\left[0, r-r_{0}\right], r_{2}=r}\left\{\frac{\frac{1}{2} r_{w}\left[W\left(r_{2}\right)^{2}-W\left(r_{1}\right)^{2}-r_{w}\right]-\int_{r_{1}}^{r_{2}} W(s) d s\left[W\left(r_{2}\right)-W\left(r_{1}\right)\right]}{r_{w}^{1 / 2}\left[r_{w} \int_{r_{1}}^{r_{2}} W(s)^{2} d s-\left(\int_{r_{1}}^{r_{2}} W(s) d s\right)^{2}\right]^{1 / 2}}\right\}
$$

\footnotetext{
${ }^{8}$ For the empirical application discussed later an augmented Dickey-Fuller specification is used with a lag order of one.
} 
where $W$ is standard Brownian motion and $r_{w}=r_{2}-r_{1}$. This limit distribution is calculated with critical values for right-sided significance testing by simulation, as described in PSY (2015a). For practical implementation, the setting for the minimum window size $r_{0}$ that is recommended in $\operatorname{PSY}(2015 \mathrm{a})$ is $0.01+1.8 / \sqrt{T}$.

\subsection{Discussion}

Somewhat unexpectedly given the right-sided nature of the PSY test (which is designed to focus on market exuberance), this procedure also has detective power against market collapse in the process (1). This property was mentioned in the introduction and has been noted in the many recent studies cited there, including the original PSY article. In what follows we provide an analytic explanation for this phenomenon.

The DF statistic is constructed from the fitted autoregressive coefficient $\rho$ in (14) and is typically employed to test hypotheses directly related to $\rho$. In particular, bubble identification is concerned with whether $\rho$ is unity (representing normal martingale market conditions) or greater than unity (representing submartingale conditions or speculative bubbles). It is, however, well known that the limit distribution of the DF statistic depends not only on the autoregressive coefficient but also on the specification of any drift term in the generating mechanism. Phillips et al. (2014) consider a martingale process of the form (13) with a parameterized drift process that enables several useful asymptotic variants. In particular, the limit distribution of the DF statistic takes different forms according to whether the drift parameter $\gamma>1 / 2, \gamma=1 / 2$, or $\gamma<1 / 2$ (with $\rho=1$ in all cases). Phillips et al. (2015b) show that under the alternative hypothesis of mildly explosive behavior (with zero drift), i.e.,

$$
\log P_{t}=\delta_{T} \log P_{t-1}+\varepsilon_{t}, \text { with } \delta_{T}=1+c T^{\alpha}, c>0, \text { and } \alpha \in[0,1)
$$

the DF statistic diverges to positive infinity. Within this framework, the null and alternative hypotheses may be formulated as a joint test involving both the intercept and the autoregressive coefficients such that

$$
\begin{aligned}
H_{0}: \mu & =g_{T} \text { and } \rho=1 \\
H_{1, \text { bubble }}: \mu & =0 \text { and } \rho>1 \\
H_{1, \text { crash }}: \mu & =K \text { and } \rho=1 .
\end{aligned}
$$

with $K=-E\left(L_{t}\right)<0$ from (4).

Although crash detection under the DGP (5) is formally equivalent to testing for structural breaks in the drift coefficient $\mu$, in practice the data series could potentially contain all three regimes (normal, bubble and crisis episodes in the data). In view of the effect of these regimes on the asymptotic behavior of the DF statistic, the induced PSY statistic may serve as a test mechanism for revealing both bubble and crisis regimes. This dual functionality and the real-time detective feature of the PSY procedure cannot be achieved through conventional structural break in mean tests. 


\section{$3.2 \quad$ Asymptotic Performance}

This section establishes the limit behavior of the PSY test under the data generating process (5). We consider behavior allowing for subsamples that fall within or straddle the two regimes. Suppose first that the regression window falls entirely in the normal regime, i.e., $r_{1}, r_{2} \in N$. In that event, the $D F_{r_{1}}^{r_{2}}$ statistic has the following limiting form (Phillips et al., 2015a)

$$
\frac{\frac{1}{2} r_{w}\left[W\left(r_{2}\right)^{2}-W\left(r_{1}\right)^{2}-r_{w}\right]-\int_{r_{1}}^{r_{2}} W(s) d s\left[W\left(r_{2}\right)-W\left(r_{1}\right)\right]}{r_{w}^{1 / 2}\left[r_{w} \int_{r_{1}}^{r_{2}} W(s)^{2} d s-\left(\int_{r_{1}}^{r_{2}} W(s) d s\right)^{2}\right]^{1 / 2}} .
$$

When the regression window straddles the normal and crisis regimes the limit behavior is quite different and is given in the following result.

Theorem 1 Under the generating mechanism (5) with $\varepsilon_{t}$ satisfying either Assumption $\boldsymbol{A} 1$ or $\boldsymbol{A} 2$ and $L_{t}$ satisfying (2) for either constant $L$ or $L \rightarrow \infty$, the unit root t-ratio test statistic diverges according to

$$
D F_{r_{1}}^{r_{2}}=O_{p}\left(T^{1 / 2}\right) \rightarrow+\infty,
$$

as $T \rightarrow \infty$, when $r_{1} \in N$ and $r_{2} \in C$.

The proof is given in the Appendix. Theorem 1 shows that when the recursive PSY regression proceeds from a normal regime to a crash regime, the $D F_{r_{1}}^{r_{2}}$ statistic diverges to infinity. Thus, movement of the data into a crash regime leads to a significant statistic on the right side of the distribution, thereby explaining the empirical outcomes of significant bubble tests for data that display crisis phenonomena rather than exuberance.

Next, suppose the regression window falls entirely in the collapsing regime, i.e., $r_{1}, r_{2} \in C$. In this event, the limit behavior of the $D F_{r_{1}}^{r_{2}}$ statistic is characterized in the following result.

Theorem 2 Under the generating mechanism (5) with $\varepsilon_{t}$ satisfying either Assumption $\boldsymbol{A} 1$ or $A 2$ and $L_{t}$ satisfying (2) for either constant $L$ or $L \rightarrow \infty$, the unit root t-ratio test statistic $D F_{r_{1}}^{r_{2}}$ converges weakly to the following limiting form

$$
\frac{2 \int_{r_{1}}^{r_{2}} W(s) d s-r_{w}\left[W\left(r_{1}\right)+W\left(r_{2}\right)\right]}{\left(\int_{0}^{r_{w}} s^{2} d s\right)^{-1 / 2}} .
$$

when $r_{1}, r_{2} \in C$.

The derivation of (19) follows directly from the results given in the Appendix and is omitted for brevity. Note that the limit distribution given by (19) is slightly different from that of the case where $\epsilon \rightarrow-1$, which corresponds to the case of constant drift, which is studied in Phillips et al. (2014).

The limit results given in (17) and (19) contrast with that of (18) given in Theorem 1, where the test statistic diverges to positive infinity. This difference suggests that the divergence in the statistic is precipitated by the structural break that occurs at $T_{c}$ as the data passes from a 
normal regime with $r_{1} \in N$ to a crisis regime with $r_{2} \in C .{ }^{9}$ More specifically, the structural break leads to a difference in the order of magnitude of the components of the test statistic. The denominator of the statistic (15) is of the same order of magnitude for the two cases of $r_{1}, r_{2} \in C$ and $r_{1} \in N$ and $r_{2} \in C$. But the numerator of the statistic has higher order magnitude when the data straddle the regimes, i.e., for the case with $r_{1} \in N$ and $r_{2} \in C$. Thus, when $r_{1}, r_{2} \in C$, we find that

$$
\begin{aligned}
\sum_{j=T_{1}}^{T_{2}} \tilde{X}_{j-1}\left(\tilde{X}_{j}-\tilde{X}_{j-1}\right) & =\sum_{j=T_{1}}^{T_{2}} \tilde{X}_{j-1} \varepsilon_{j}-\sum_{j=T_{1}}^{T_{2}} \tilde{X}_{j-1} L_{j} \\
& =\sum_{j=T_{1}}^{T_{2}} \tilde{X}_{j-1} \varepsilon_{j}\left\{1+o_{p}(1)\right\}=O_{p}\left(T^{3 / 2}\right),
\end{aligned}
$$

since $\sum_{j=T_{1}}^{T_{2}} \tilde{X}_{j-1} L_{j}=o_{p}(1),{ }^{10}$ whereas when $r_{1} \in N$ and $r_{2} \in C$

$$
\begin{aligned}
\sum_{j=T_{1}}^{T_{2}} \tilde{X}_{j-1}\left(\tilde{X}_{j}-\tilde{X}_{j-1}\right) & =\sum_{j=T_{1}}^{T_{2}} \tilde{X}_{j-1} \varepsilon_{j}-\sum_{j=T_{c}+2}^{T_{2}} \tilde{X}_{j-1} L_{j} \\
& =-\sum_{j=T_{c}+2}^{T_{2}} \tilde{X}_{j-1} L_{j}\left\{1+o_{p}(1)\right\}=O_{p}\left(T^{2}\right) \rightarrow+\infty
\end{aligned}
$$

assuring divergence of the test statistic to positive infinity.

Finally, we compare these results with the case studied in earlier work (Phillips and Shi, 2018, 2017) where the data follows a mildly integrated process during the crisis regime.

Theorem 3 Under the generating mechanism (12) with $\varepsilon_{t}$ satisfying either Assumption $\boldsymbol{A} 1$ or $\boldsymbol{A} 2$, the unit root t-ratio test statistic $D F_{r_{1}}^{r_{2}}=O_{p}\left(T^{1 / 2}\right) \rightarrow-\infty$ as $T \rightarrow \infty$, when $r_{1} \in N$ and $r_{2} \in C$.

Theorem 3 gives the limit behavior of the DF statistic under the DGP (12). The proof follows directly as in Phillips and Shi $(2018,2017)$ and is available from the authors upon request. Thus, under the generating mechanism used in PSY and PS, the unit root test diverges to negative infinity as the sample size goes to infinity and therefore appears to have no discriminatory power in terms of the right-sided testing environment associated with bubble detection. This result contrasts sharply with Theorem 1 where the same test statistic has strong discriminatory power

\footnotetext{
${ }^{9}$ It can also be shown that the DF statistic diverges to positive infinity when $r_{1} \in N$ and $r_{2}$ falls in a regime governed by a deterministic linear trend process such as $\log P_{t}=g+\log P_{t-1}+\varepsilon_{t}$ with constant $g<0$ or $\log P_{t}=c_{1} T^{-\alpha}+\log P_{t-1}+\varepsilon_{t}$ with $c_{1}<0$ and $\left.\alpha<1 / 2\right)$. Nevertheless, unlike such models with deterministic linear trends, the random drift martingale process permits a negative drift with an embedded variance inflation and hence can generate more realistic patterns of market collapse. It is therefore preferred as a model for financial crises.

${ }^{10}$ Note that when $\epsilon \rightarrow-1$ (i.e., $L_{j}=L$ ) and $r_{1}, r_{2} \in C, \sum_{j=T_{1}}^{T_{2}} \tilde{X}_{j-1} L_{j}=L \sum_{j=T_{1}}^{T_{2}} \tilde{X}_{j-1}=0$ by construction.
} 
in right-sided testing. The difference arises because under DGP (12) the numerator

$$
\sum_{j=T_{1}}^{T_{2}} \tilde{X}_{j-1}\left(\tilde{X}_{j}-\tilde{X}_{j-1}\right)=\left(\gamma_{T}-1\right) \sum_{j=T_{c}}^{T_{2}} \tilde{X}_{j-1}^{2}\left\{1+o_{p}(1)\right\}=O_{p}\left(T^{2-\beta}\right) \rightarrow-\infty
$$

which assures divergence to negative infinity.

By virtue of its construction from unit root statistics, the backward sup statistic BSDF also diverges to positive infinity under (5) and to negative infinity under (12) at the same rate $T^{1 / 2}$, in both cases when the observation interval includes the crisis regime (i.e. for $r_{2} \in C$ ). This differing behavior of the BSDF statistic determines the outcome of the PSY detection process (designed originally for bubbles in the expansionary phase) under the two different crisis mechanisms.

To fix ideas, let $c v_{r}\left(\beta_{T}\right)$ be the $\left(1-\beta_{T}\right) 100 \%$ percentile of the limit distribution under the null (16) (i.e., the critical value of the $B S D F_{r}$ statistic) and assume that $c v_{r}\left(\beta_{T}\right) \rightarrow \infty$ as $\beta_{T} \rightarrow 0$. The break date $T_{c}$ is estimated as the first chronological observation whose BSDF statistic exceeds $c v_{r}\left(\beta_{T}\right)$.

Theorem 4 Under the data generating process (5) with $\varepsilon_{t}$ satisfying either Assumption $\boldsymbol{A} 1$ or $\boldsymbol{A} 2$ and $L_{t}$ satisfying (2) for either constant $L$ or $L \rightarrow \infty$, the break date $r_{c}$ of crash origination is consistently estimated by the PSY detector if

$$
\frac{1}{c v_{r}\left(\beta_{T}\right)}+\frac{c v_{r}\left(\beta_{T}\right)}{T^{1 / 2}} \rightarrow 0
$$

On the other hand, the PSY procedure cannot consistently estimate the break date $r_{c}$ under the $D G P(12)$.

The proof of Theorem 4 is based on Theorems 1 and 3 and follows directly as in the arguments given in PSY (2015b).

Suppose the normal regime in (5) is stationary (e.g., $\log P_{t}=\delta \log P_{t-1}+\varepsilon_{t}$, with $0<\delta<1$ ). It can be shown that the BSDF statistic for the case of $r_{1} \in N$ and $r_{2} \in C$ will again diverge to positive infinity. This is in sharp contrast to the limiting distribution under the null (17) and the case where both $r_{1}$ and $r_{2}$ are in the stationary normal regime. Therefore, the right-sided PSY test will still be able to consistently estimate the break date in this case.

Given the substantial empirical evidence that now exists showing that the PSY algorithm detects crisis episodes, particularly abrupt collapses or slow sustained collapses, these analytic results indicate that the generating process (5) has a considerable advantage over the mildly integrated mechanism (12) in modeling various collapse processes that arise in practical work with financial and economic data.

\subsection{Finite Sample Performance}

This section provides simulation evidence that explores the properties of the PSY procedure under a market collapse generating mechanism based on the $L$ - process model described above. The data generating processes are (5) in Table 1 for logarithmic prices and (9) in Table 2 for 
bond yields, with parameter settings the same as for Figures $1 \mathrm{~b}$ and $2 \mathrm{~b}$. The number of replications is 5,000 . We vary some of the key parameters to gain a more comprehensive view of test performance.

The performance of the PSY procedure for crisis detection is compared with the CUSUM monitoring strategy for structural breaks. We apply the CUSUM strategy with two different model specifications: levels and first differences. Suppose the regression model is $z_{t}=\beta^{\prime} x_{t}+\varepsilon_{t}$, where $\varepsilon_{t}$ is the error term and $\beta$ is either a $k \times 1$ row vector or a scalar. We have $z_{t}=y_{t}$ and $x_{t}=$ $\left[1, y_{t-1}\right]^{\prime}$ for the level specification and $z_{t}=\Delta y_{t}$ and $x_{t}=1$ for the first difference specification. We test for the stability of both the intercept and the first order autoregressive coefficients with the level specification. On the other hand, when employing the difference specification we impose a unit root structure while testing for the stability of the drift coefficient. The training period of the CUSUM strategy contains $m$ observations, which is assumed to be the same as the minimum window size for the PSY strategy. The monitoring starts from the $(m+1)^{t h}$ observation. Let $\hat{\beta}^{(i)}$ be the OLS estimate obtained from the sample running from the first to the $i^{\text {th }}$ observation. The test statistic is defined as

$$
Q_{t}^{m}=\hat{\sigma} \sum_{i=m+1}^{t} \omega_{i} \text { with } \omega_{i}=\hat{\varepsilon}_{i} / v_{i}^{1 / 2}
$$

where $\hat{\varepsilon}_{i}=z_{i}-\hat{\beta}^{(i-1)} x_{i}, v_{i}=1+x_{i}^{\prime}\left(\sum_{j=1}^{i-1} x_{j} x_{j}^{\prime}\right)^{-1} x_{i}$ and $\hat{\sigma}=\frac{1}{m-k} \sum_{j=1}^{m}\left(z_{j}-\beta^{(m)} x_{j}\right)^{2}$. Chu et al. (1996) show that under the null hypothesis of parameter stability

$$
\lim _{m \rightarrow \infty} P\left\{\left|Q_{t}^{m}\right| \geq \sqrt{t+m-k}\left[a^{2}+\ln \left(\frac{t+m-k}{m-k}\right)\right]^{1 / 2}\right\}=\exp \left(-a^{2} / 2\right),
$$

where $a^{2}=6$ for $5 \%$ asymptotic size.

We report the successful detection rate (SDR) and mean and standard deviation of the bias of the estimated crash origination date, i.e. $\hat{r}_{c}-r_{c}$. The successful detection rate provides a more complete performance measure than power. Successful detection satisfies the following two conditions: (i) for all $r \in\left[r_{0}, 1\right]$, there should be at least one realized test statistic $B S D F_{r}\left(r_{0}\right)$ that exceeds the relevant critical value; and (ii) the estimated crash origination date $\hat{r}_{c}$ should exceed the actual date $r_{c}$ of collapse. Note that only the first condition is required for power calculation.

It is obvious from both tables that for all three tests power declines as the break point moves towards the end of the sample period. This decline in power occurs because there are fewer observations in the crisis regime as $r_{c}$ moves towards unity and therefore the crisis signal is weaker given the same collapse intensity. The successful detection rates of all tests increase with the sample size, which is expected given test consistency. For example under (5), the SDR of the PSY test rises from $49 \%$ to $88 \%$ when $T$ increases from 50 to 200 . The last panels of Tables 1 and 2 show the SDRs of the tests with respect to variations in collapse intensity $L$. As expected and as evident in the tables, power increases with $L$.

The estimation accuracy of the origination date of all tests remains roughly the same with respect to sample size $T$ and collapse magnitude $L$. However, bias reduces as the break point 
Table 1: The successful detection rate of the BSDF test for crisis identification under DGP (5) for log prices.

\begin{tabular}{|c|c|c|c|c|c|c|}
\hline & \multicolumn{2}{|c|}{ BSDF } & \multicolumn{2}{|c|}{ CUSUM (level) } & \multicolumn{2}{|c|}{ CUSUM (difference) } \\
\hline & SDR & $\hat{r}_{c}-r_{c}$ & SDR & $\hat{r}_{c}-r_{c}$ & SDR & $\hat{r}_{c}-r_{c}$ \\
\hline \multicolumn{7}{|c|}{$T=50$ and $L=0.17$} \\
\hline$r_{c}=0.6$ & 0.74 & $0.20(0.11)$ & 0.72 & $0.21(0.10)$ & 0.76 & $0.20(0.10)$ \\
\hline$r_{c}=0.7$ & 0.63 & $0.15(0.08)$ & 0.55 & $0.18(0.08)$ & 0.64 & $0.17(0.07)$ \\
\hline$r_{c}=0.8$ & 0.49 & $0.11(0.05)$ & 0.31 & $0.13(0.05)$ & 0.41 & $0.12(0.05)$ \\
\hline$r_{c}=0.9$ & 0.29 & $0.06(0.03)$ & 0.10 & $0.07(0.03)$ & 0.16 & $0.07(0.03)$ \\
\hline \multicolumn{7}{|c|}{$r_{c}=0.8$ and $L=0.17$} \\
\hline$T=50$ & 0.49 & $0.11(0.05)$ & 0.31 & $0.13(0.05)$ & 0.41 & $0.12(0.05)$ \\
\hline$T=100$ & 0.70 & $0.10(0.05)$ & 0.44 & $0.13(0.05)$ & 0.60 & $0.12(0.05)$ \\
\hline$T=150$ & 0.81 & $0.10(0.05)$ & 0.58 & $0.13(0.04)$ & 0.72 & $0.12(0.05)$ \\
\hline$T=200$ & 0.88 & $0.10(0.05)$ & 0.71 & $0.13(0.04)$ & 0.82 & $0.11(0.05)$ \\
\hline \multicolumn{7}{|c|}{$T=50$ and $r_{c}=0.8$} \\
\hline$L=0.1$ & 0.39 & $0.11(0.06)$ & 0.10 & $0.14(0.06)$ & 0.17 & $0.14(0.05)$ \\
\hline$L=0.2$ & 0.54 & $0.11(0.05)$ & 0.42 & $0.13(0.05)$ & 0.49 & $0.12(0.05)$ \\
\hline$L=0.3$ & 0.62 & $0.10(0.05)$ & 0.69 & $0.11(0.05)$ & 0.70 & $0.10(0.05)$ \\
\hline$L=0.4$ & 0.65 & $0.09(0.05)$ & 0.82 & $0.09(0.05)$ & 0.80 & $0.09(0.05)$ \\
\hline
\end{tabular}

moves towards the end of the sample period. The latter is due to the definition of the successful detection rate which requires that only those estimates greater than $r_{c}$ are counted, thereby ensuring that the maximum bias is constrained to be $1-r_{c}$.

In general, the PSY procedure outperforms the CUSUM strategy (both for levels and for first differences), except when the magnitude of the crash is extremely large. The superiority of the PSY strategy is obvious in Table 1. In Table 2, the SDR of the CUSUM strategy exceeds that of the PSY strategy when $L=391.79$ (the top panel) or when $L=400$ (the last row). The relative performance of the two CUSUM strategies varies with the parameter settings.

\section{Detecting the Subprime Mortgage Crisis}

The bursting of the US real estate bubble in 2007 led to mortgage delinquencies and foreclosures coupled with a major secondary impact on financial markets through the devaluation of housingrelated securities. This financial market impact triggered instability in the global financial system and an economic recession in the US. As depicted in Figure 1a, log prices of S\&P index stocks dropped dramatically starting from late 2007 towards the end of the sample period in 2009 . We apply the PSY procedure to both the log prices and the price-dividend ratio of the monthly S\&P500 index over the period from January 2005 to March 2009. The 95\% finite sample critical values are obtained by a wild bootstrap procedure. The wild bootstrap is used to replicate the pattern of heteroskedasticity in the original shocks of the financial return data and hence is expected to reduce the risk of any size distortion in practical application of the PSY procedure 
Table 2: The successful detection rate of the BSDF test for crisis identification under DGP (9) for bond yields.

\begin{tabular}{|c|c|c|c|c|c|c|}
\hline & \multicolumn{2}{|c|}{ BSDF } & \multicolumn{2}{|c|}{ CUSUM (level) } & \multicolumn{2}{|c|}{ CUSUM (difference) } \\
\hline & SDR & $\hat{r}_{c}-r_{c}$ & SDR & $\hat{r}_{c}-r_{c}$ & SDR & $\hat{r}_{c}-r_{c}$ \\
\hline & \multicolumn{6}{|c|}{$T=50$ and $L=391.79$} \\
\hline$r_{c}=0.6$ & 0.84 & $0.16(0.10)$ & 0.93 & $0.15(0.10)$ & 0.92 & $0.11(0.08)$ \\
\hline$r_{c}=0.7$ & 0.80 & $0.12(0.07)$ & 0.88 & $0.11(0.07)$ & 0.93 & $0.10(0.07)$ \\
\hline$r_{c}=0.8$ & 0.71 & $0.09(0.05)$ & 0.79 & $0.09(0.05)$ & 0.86 & $0.09(0.05)$ \\
\hline$r_{c}=0.9$ & 0.52 & $0.05(0.03)$ & 0.60 & $0.05(0.03)$ & 0.60 & $0.06(0.03)$ \\
\hline \multicolumn{7}{|c|}{$r_{c}=0.8$ and $L=100$} \\
\hline$T=50$ & 0.69 & $0.09(0.05)$ & 0.18 & $0.11(0.05)$ & 0.12 & $0.14(0.05)$ \\
\hline$T=100$ & 0.80 & $0.08(0.05)$ & 0.26 & $0.10(0.05)$ & 0.20 & $0.14(0.05)$ \\
\hline$T=150$ & 0.83 & $0.07(0.05)$ & 0.31 & $0.10(0.05)$ & 0.29 & $0.14(0.05)$ \\
\hline$T=200$ & 0.81 & $0.06(0.05)$ & 0.38 & $0.10(0.05)$ & 0.39 & $0.14(0.04)$ \\
\hline \multicolumn{7}{|c|}{$T=50$ and $r_{c}=0.8$} \\
\hline$L=10$ & 0.27 & $0.11(0.06)$ & 0.03 & $0.11(0.05)$ & 0.01 & $0.09(0.06)$ \\
\hline$L=100$ & 0.70 & $0.09(0.05)$ & 0.18 & $0.11(0.05)$ & 0.12 & $0.14(0.05)$ \\
\hline$L=200$ & 0.71 & $0.09(0.05)$ & 0.47 & $0.10(0.05)$ & 0.55 & $0.12(0.05)$ \\
\hline$L=400$ & 0.71 & $0.09(0.05)$ & 0.79 & $0.08(0.05)$ & 0.86 & $0.09(0.05)$ \\
\hline
\end{tabular}

(Harvey et al., 2016).

Figure 3: The logarithmic price (left) and the price-dividend ratio (right) of the S\&P 500 price index running from January 2005 to March 2009. The shaded areas are the identified periods of market crashes based on the PSY dating procedure.

(a) The log S\&P 500 price index

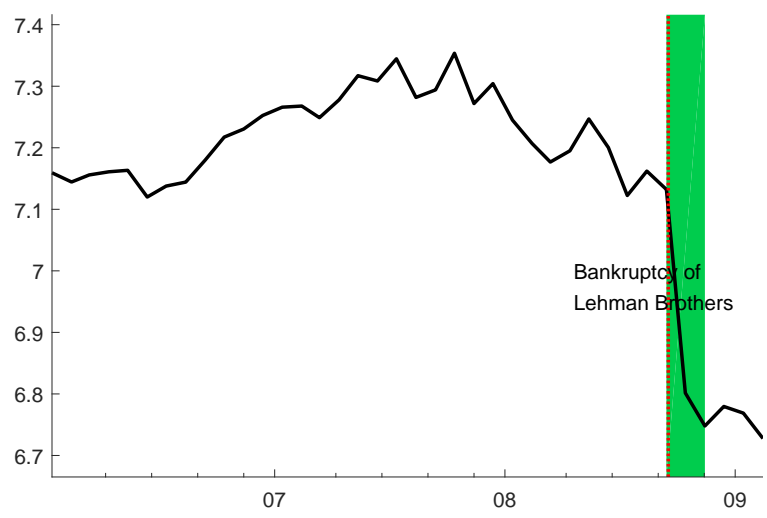

(b) The price-dividend ratio of the S\&P500 index

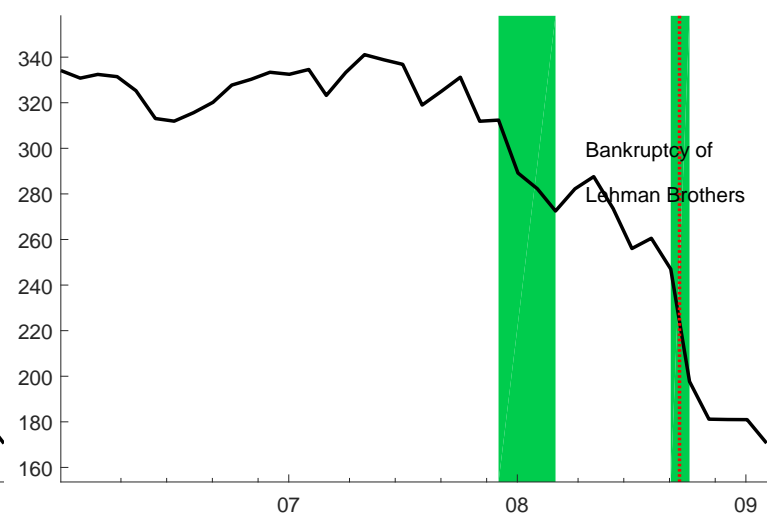

Figure 3 plots the log price (left panel) and the price-dividend ratio (right panel) of the S\&P 500 index. The shaded areas are the identified periods of the market crash based on the 
PSY detection algorithm. For the log price series, the crisis monitoring algorithm finds a single episode of market collapse. The identified origination date coincides with the collapse of Lehman Brothers in September 2008. By comparison, the price-dividend ratio series appears to contain more sensitive information concerning the market downturn with a warning alert of a crash in early 2008. With this series, the PSY procedure detects evidence of the emergent crisis much earlier after the market peak in 2007, revealing an initial episode of collapse that runs from January to March 2008, followed by the later collapse in September 2008 at the time of the Lehman Brothers bankruptcy.

For both series, this application demonstrates that the right-sided PSY test and market monitoring procedures are useful in detecting and dating market crashes as well as episodes of market exuberance and bubbles, confirming the relevance of the limit theory given earlier for the detection of collapse episodes in financial data.

\section{$5 \quad$ Detecting the Sovereign Debt Crisis}

We focus on the sovereign debt crisis experienced in the European Union (EU) following the global financial crisis. The countries included in the study are Portugal, Ireland, Italy, Greece, and Spain (PIIGS). These are the five EU member states that were unable to refinance their government debt or to bail out over-indebted banks on their own during the debt crisis. We measure the sovereign credit risk with both 10-year government bond yields and five-year credit default swaps (CDS).

\subsection{Government Bond Yield Indicators}

The monthly 10-year government bond yield spreads of the PIIGS countries (along with Germany) from January 2001 to September 2016 are plotted in Figure 4. The data are obtained from DataStream.

As is evident in the figure, government yields in these five countries over the period 2003 to late 2008 move almost in line with each other with only mild fluctuations in their levels around $5 \%$. The bond yields of Greece and Ireland rose after the bailout announcement of the Irish government on September 30, 2008 and reached a temporary peak in January 2009. A second round of far more aggressive expansion in bond yields started soon after the Greek government's announcement of a 300 billion Euro debt on December 10, 2009. Led by the rapid and substantial rise in Greek yields to over $25 \%$, the yields of all countries continued to mount to historical highs, with Greek yields clearly dominating in terms of the magnitude of this yield inflation. The Greek government bond yield peaked in March 2012 when Greece defaulted on its debt.

Portugese and Irish debt yields followed similar time paths peaking in January 2012 (Portugal) and July 2011 (Ireland). A less dramatic rise in Greek government bond yields occurred over the period from late 2014 to early 2015 with some later fluctuations in levels through 2016 . This subsequent episode was triggered by the sustained economic recession through to the fourth quarter of 2014, a dramatic one-day fall of $12 \%$ in the Athens stock market in December 2014, and the election victory of the political party Syrizia, which led the new Greek government to 
Figure 4: The 10-year government bond yield (\%) of PIIGS from January 2001 to September 2016

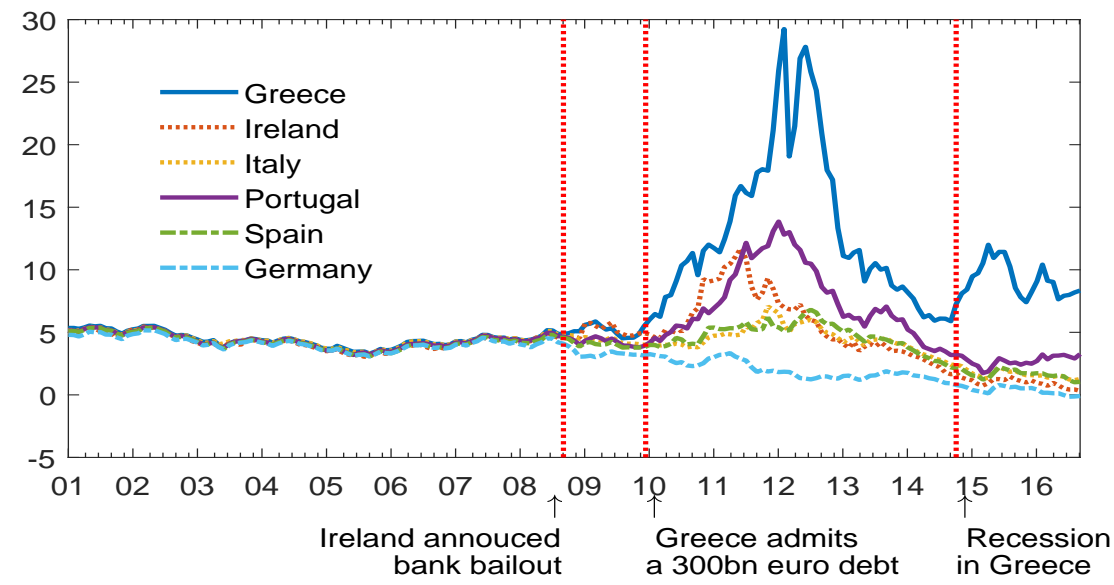

reject the existing bailout terms. In response, the European 'Troika'11 temporarily suspended all scheduled remaining aid to Greece. The yields of Spain and Italy closely follow each other during this period. In the aftermath of the crisis, the debt yields of all the PIIGS countries (except Greece) declined from mid 2015 onwards and have been relatively stable since. The German government bond yields remained uniformly the lowest throughout the entire period and with a minor fluctuation in early 2015 descended over the full tenure of the crisis, consistent with the perception of a 'flight-to-quality' in government debt (Beber et al., 2009).

In our empirical work with these data, we applied the PSY test to both the bond yields and the bond yield spread between the PIIGS and Germany. In the latter case, the German bond yield serves as a proxy 'fundamental', capturing changes in macroeconomic conditions and investor risk aversion. The wild bootstrap is employed to obtain appropriate $95 \%$ finite-sample critical values for the test. The PSY test statistic sequence starts from February 2003. Figure 5 plots the 10-year government bond yields (left panel (a)) and bond yield spreads between the PIIGS countries and Germany (right panel (b)), with the shaded areas in the figure showing the crisis episodes identified by the PSY procedure. The first vertical line indicates the date of the Irish government's announcement of bank bailout packages in Setember 2008; the second vertical line indicates the date when the Greek government admitted a 300 billion Euro debt; and the final line marks the beginning of the 2014 economic recession in Greece.

Based on the 10-year government bond yield series, the PSY procedure finds no evidence of a market crash in 2007-2008 in the PIIGS countries. This result contrasts with the findings for bond yield spreads, where prolonged periods of deviation from martingale dynamics are detected for all countries. This suggests that bond yield spreads provide a much stronger signal concerning sovereign risk than bond yield levels. German government bonds were seen as a 'safe haven' during

\footnotetext{
${ }^{11}$ The Troika includes the European Commission, the European Central Bank, and the International Monetary Fund.
} 
Figure 5: The PSY test based on the 10-year government bond yields (left) and the bond yield spreads (right) of the PIIGS countries from February 2003 to September 2016. The shaded areas are the identified periods of market crashes based on the procedure.

(a) PSY test based on bond yields
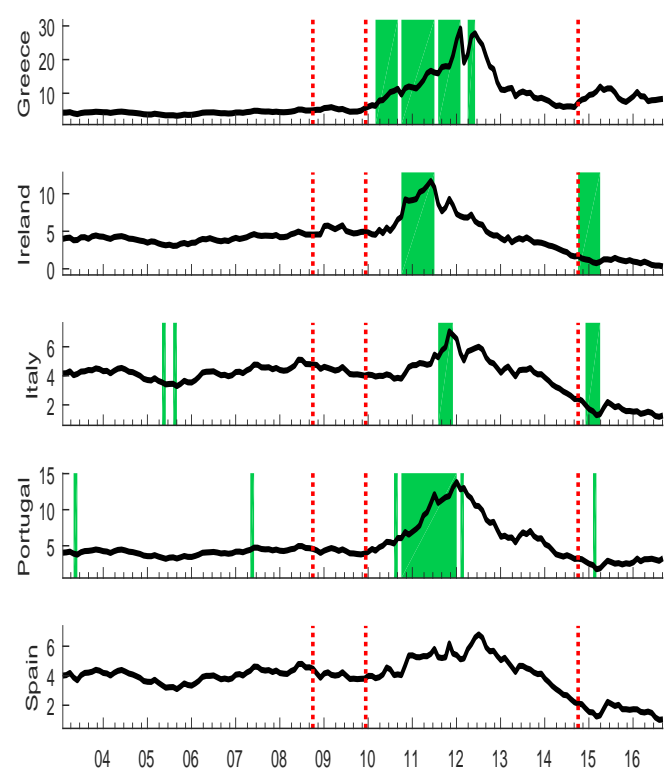

(b) PSY test based on bond yield spreads between the PIIGS countries and Germany
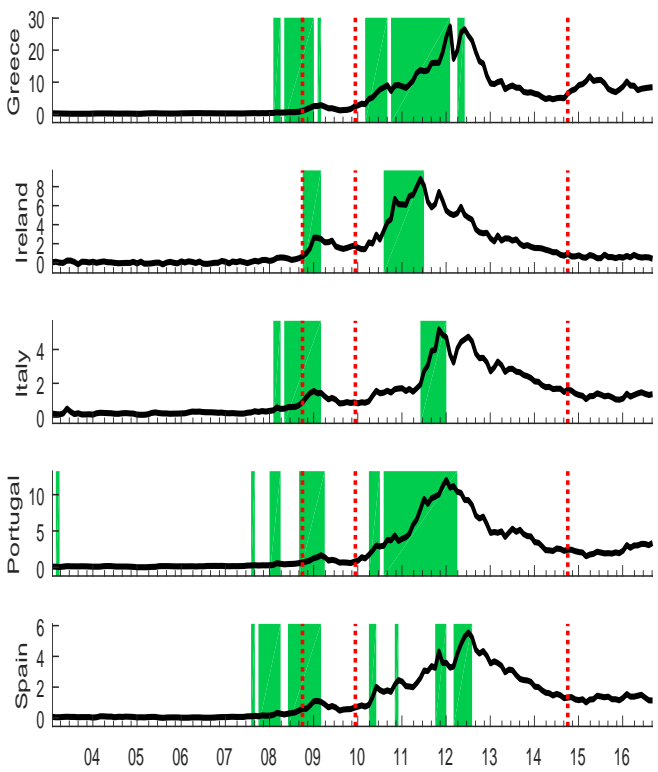

the debt crisis and the increased demand during this period for German bonds led to lower bond yields. The spreads reflect relative movements in both yield series, thereby providing a measure of relative sovereign risk and providing a more sensitive ongoing indicator of the prevailing crisis conditions.

Based on the bond yield spreads, crashes are detected in every government bond market during the global financial crisis. The warning signal in Ireland switched on immediately after Ireland's announcement of bailout packages for the financial sector. This finding is consistent with the view that the fiscal intervention by government transferred financial sector stress to the public sector (Mody (2009); Zoli and Sgherri (2009); Ejsing and Lemke (2011); Acharya et al. (2014)). The timing of the warning signals differ for the other PIIGS countries. While the bailout packages for the financial sectors were announced at the end of 2008 for those countries, the test results show that the sovereign bond yields of Greece, Italy, Portugal and Spain all began to deviate from the German yields in late 2007 or early 2008. So, the warning alerts in the data are evident much earlier for these countries indicating that market assessment of sovereign risk predated the bailout announcements and coincided with recognition of more general global financial crisis conditions that affected EU countries. Termination of this crisis episode occurs around the 
same time in all PIIGS countries in early 2009.

The second episode of major deviations in yield spreads occurs with the emergence of the European debt crisis. The episode began soon after Greece's announcement of a $€ 300$ billion debt and it affected Greece, Portugal, Spain and Ireland in early-to-mid 2010. The crisis intensified in 2011, involving all PIIGS countries. Greece suffered the longest duration of this debt crisis, followed by Portugal and Ireland. Spain appeared to suffer the least and the impact there was intermittent. The warning signals switched off in late 2011 in Ireland, early 2012 in Italy, Portugal and Greece, and by mid-2012 in Spain.

\subsection{Credit Default Swap Indicators}

A credit default swap (CDS) is a financial swap agreement in which the buyer of the CDS contract pays a periodic fee to the seller until the contract matures or a certain specified credit event occurs. The periodic payments are usually written as a percentage of the underlying asset's notional value and constitute the CDS premium. In the event of a loan default or other credit event, the CDS seller compensates the buyer with the difference between the market value and face value of the issue. The 'restructuring clause' of a CDS contract specifies the credit events that trigger settlement. The CDS contract fee is paid semi-annually on an actual/360 day count basis and therefore the contracts do not suffer from declining maturities like conventional debt instruments. By non-arbitrage theory, the CDS premium of sovereign risk should equal the spread between the government bond yield and the risk free rate. ${ }^{12}$

The daily CDS premiums are sourced from the Markit database, which is an average across all quotes provided by market makers after a series of data cleaning tests. We focus on the spreads of all the CDS contracts (denominated in US dollars) with the full restructuring clause (the 2003 ISDA Definition). ${ }^{13}$ We use the five-year spreads because these contracts are the most liquid and account for a large proportion of the CDS market. Unlike the five-year CDS contracts, the 10-year government bonds are much less liquid. Hence bond spreads bear both the credit risk and the liquidity risk especially during periods of market stress (Beber et al. (2009), Manganelli and Wolswijk (2009), and Schwarz (2017)). The CDS premium measures market credit risk and serves here as an alternative proxy for sovereign risk.

Figure 6 displays the CDS premiums of the PIIGS countries, along with Germany, for the sample period running from $01 / 01 / 2007$ to $02 / 09 / 2016$. The upper panel is for Greece and the lower panel for all the other countries. The dynamic patterns of the CDS premia are similar to the 10-year government bond yields (Figure 4). It is noteworthy that the Greek CDS premium at the peak of the European debt crisis is more than ten times that of other countries. The Greek CDS market was closed in March 2012 when Greece defaulted on its debt. The CDS premium reached 231 percent at the time of market closing. The market reopened in June 2013 with a premium around 10 percent and was followed by another small market bump in the first half of 2015.

\footnotetext{
${ }^{12}$ See, for example, Acharya et al. (2002) and Hull and White (2000).

${ }^{13}$ Under this contract option, any restructuring event qualifies as a credit event (and any bond of maturity up to 30 years is deliverable).
} 
Figure 6: The CDS premium (\%) of the PIIGS countries sampled from 01/01/2007 to 02/09/2016.
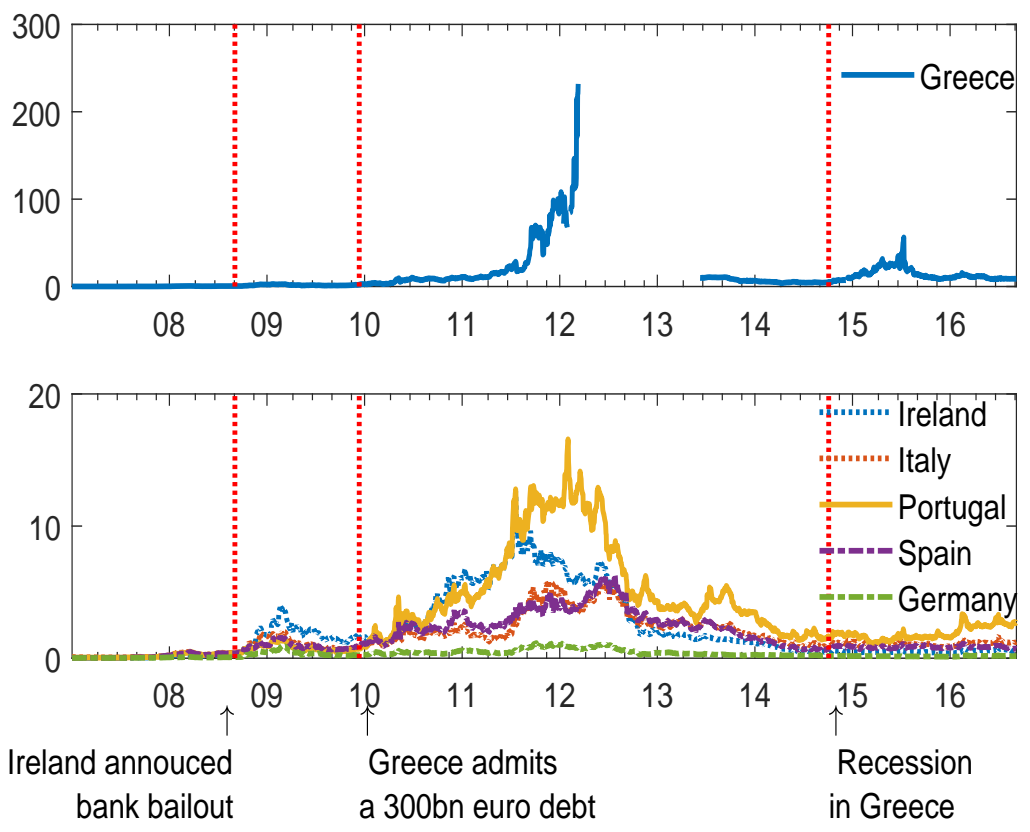

The PSY test with wild bootstrapping is applied to the spreads between the sovereign and Germany CDS premia. The PSY statistic starts from 08/06/2007. ${ }^{14}$ Figure $7 \mathrm{~b}$ plots the CDS premium of the PIIGS countries, with the shaded areas being the identified crash periods. The PSY test results based on the bond yield spread are plots in Figure 7a for comparison.

As with the bond yield spreads, the PSY test based on CDS spreads finds two major periods of upward expansions across all countries over the sample period, namely the periods around the subprime mortgage crisis in 2007-2009 and the European debt crisis in 2010-2012. Interestingly, using information from the CDS market, one would be able to detect ballooning sovereign risk in 2007-2009 earlier for all countries than from the bond market. In particular, the sovereign risk in Ireland was detected one year earlier in the CDS market (October 2007) than in the bond market. For the European debt crisis period in 2010-2012, signals from the bond market are stronger than those from the CDS market. One potential explanation is that liquidity risk, in addition to credit risk, is a major concern during this period. The concern is less severe for CDSs than bonds, given that the CDS market is much more liquid. This results in faster expansions in bond yields than in CDS premiums and hence the signal of sovereign risk is stronger in the bond market. ${ }^{15}$

\footnotetext{
${ }^{14}$ For the Greek market, we re-initiate the test in June 2013, with the minimum window size kept the same.

${ }^{15}$ Under the 2003 ISDA, a re-denomination (in which countries leave the European Monetary Union and reintroduce their own national currencies) is less likely to be classified as a CDS credit event and hence the CDS data may not reflect this systemic risk accurately. However, we believe the difference between the results for the bond yield and CDS spreads cannot be explained by the re-denomination risk. This is because there is no obvious difference in the PSY test results between the bond yield spreads and the CDS spreads for Italy and Spain over the period from 2014-2016, while the re-denomination risk is believed to have affected these two countries the most over the
} 
Figure 7: The PSY test based on the 10-year government bong yield spreads (left) and the credit default swap premium spreads (right) between PIIGS countries and Germany from January 2007 to September 2016. The shaded areas are the periods identified using the procedure.

(a) PSY test based on bond yield spreads
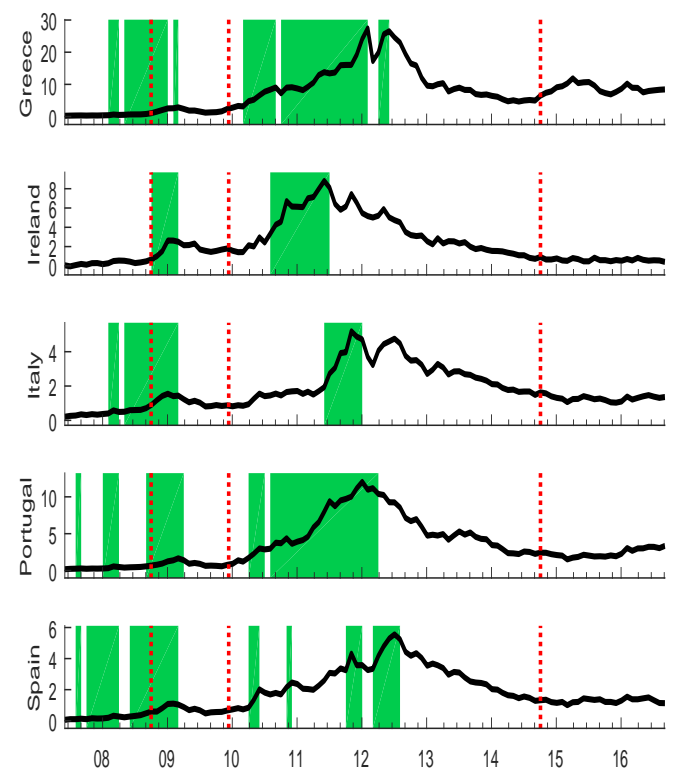

(b) PSY test based on CDS spreads
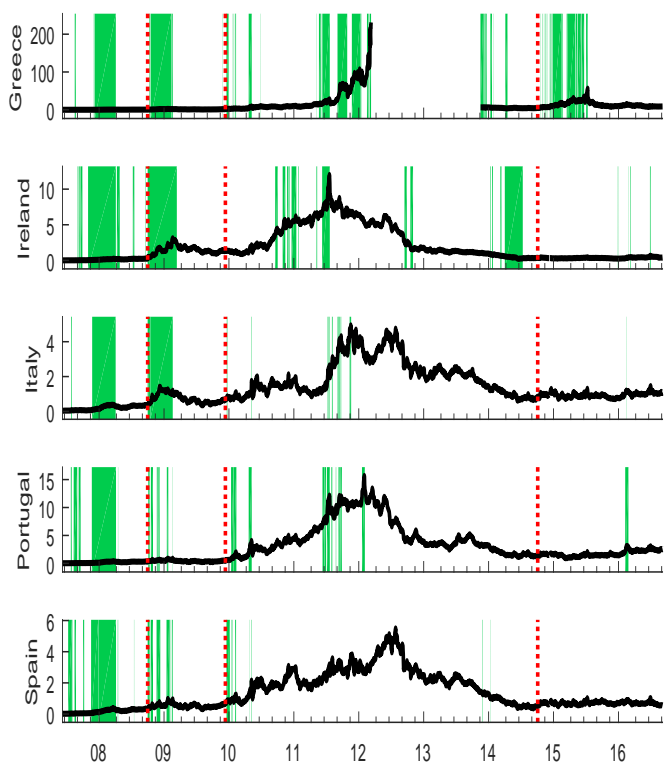

Based on the CDS spreads, the PSY procedure detects an additional episode of sovereign risk in Greece which starts from the last quarter of 2014. This period overlaps with the latest economic recession in Greece which we described earlier. Notice that we do not detect this episode with the bond yield spreads.

\section{Conclusions}

This paper introduces a new data generating mechanism for modeling financial market crises and collapses. The model involves an asymmetrically distributed input process that raises the probability of sustained, but not necessarily monotonic, market movements in one direction, thereby accommodating both crisis and expansionary phases. The new model is particularly suited to capturing abrupt market falls. It accords with market crash definitions used in finance theory such as Gennotte and Leland (1990) and Barlevy and Veronesi (2003) and it can explain the upward expansionary dynamics of bond yields during crisis periods. Both asymptotic properties and finite sample performance reveal that the recursive and moving window procedures of PSY recent period (De Santis, 2015; Nolan, 2018). 
(2015a,b) have detective capability for market meltdowns as well as expansions, providing analytic support to earlier evidence of this capability that has arisen in various empirical studies.

As an illustration of this capacity to identify sharp market downturns, the PSY procedure was applied to S\&P 500 stock market data from January 2005 to March 2009. The test switches on a warning signal in January 2008 (lasting for three months) and again upon the Lehman Brothers bankruptcy in September 2008, capturing key discontinuities in the financial environment during the GFC.

The effectiveness of the procedure for monitoring ballooning sovereign risk was illustrated with an application to Portugal, Ireland, Italy, Greece, and Spain. This analysis of European Union sovereign risk spreads reveals two common crisis episodes for the PIIGS countries over 2001-2016, using 10-year government bond yield spreads and 5-year credit default swap spreads. The warning alert given by the test predates the EU bailout of the financial sector in September 2008, switching on as early as July 2007 for Portugal and for Spain with the CDS spread data or, alternately, September 2007 with the bond yield spread data. Subsequently, the test dates origination of the European debt crisis to December 2009 from the CDS market, soon after the Greek government's announcement of $€ 300$ billion indebtedness. Interestingly, the origination date for the debt crisis is evidenced from the bond market data much later in April 2011. The CDS data also signals the 2014-2015 crisis episode in Greece that was triggered by the economic recession.

These illustrations show that whilst the 'true' generating mechanisms of financial time series during market meltdowns and expansions are unknown, most likely unknowable, and inevitably alter from one crisis to another, it is possible to capture the effects of major disturbances in the financial environment using recursive test procedures that are sensitive to departures from martingale assumptions in both expansionary and collapse directions. The reduced form model we use for price dynamics during market crashes allows for a mixture of market responses that produces realistic patterns of price behavior during market reversals and can be viewed as a plausible modeling mechanism for data generated during a variety of different collapse scenarios. Asymptotic theory and finite sample evidence from this econometric model as well as the empirical illustrations together show the potential of these recursive monitoring algorithms for detecting and dating major changes in financial environment conditions that produce market meltdowns and exuberance. ${ }^{16}$

\section{References}

Acharya, V., I. Drechsler, and P. Schnabl (2014). A pyrrhic victory? Bank bailouts and sovereign credit risk. The Journal of Finance 69, 2689-2739.

Acharya, V. V., S. R. Das, and R. K. Sundaram (2002). Pricing credit derivatives with rating transitions. Financial Analysts Journal 58, 28-44.

\footnotetext{
${ }^{16}$ It is worth noting that with a positively signed L-component the L-process could potentially be used to model market expansions. A future research direction in bubble emergence detection is to develop a test for transition from normal martingale behavior to such an L-process expansion compared with transition to explosive dynamics.
} 
Barlevy, G. and P. Veronesi (2003). Rational panics and stock market crashes. Journal of Economic Theory 110, 234-263.

Beber, A., M. W. Brandt, and K. A. Kavajecz (2009). Flight-to-quality or flight-to-liquidity? Evidence from the euro-area bond market. The Review of Financial Studies 22, 925-957.

Chu, C. J., M. Stinchcombe, and H. White (1996). Monitoring structural change. Econometrica $64,1045-1065$.

Cont, R. and L. Wagalath (2013). Running for the exit: Distressed selling and endogenous correlation in financial markets. Mathematical Finance 23, 718-741.

Cont, R. and L. Wagalath (2016). Fire sales forensics: Measuring endogenous risk. Mathematical Finance 26, 835-866.

Cutler, D. M., J. M. Poterba, and L. H. Summers (1989). What moves stock prices? The Journal of Portfolio Management 15, 4-12.

De Santis, R. A. (2015). A measure of redenomination risk. ECB Working Paper No. 1785. Available at SSRN: https://ssrn.com/abstract=2620732.

Deng, Y., E. Girardin, R. Joyeux, and S. Shi (2017). Did bubbles migrate from the stock to the housing market in china between 2005 and 2010? Pacific Economic Review 22, 276-292.

Dungey, M., R. Fry, B. González-Hermosillo, and V. L. Martin (2005). Empirical modelling of contagion: A review of methodologies. Quantitative Finance 5, 9-24.

Ejsing, J. and W. Lemke (2011). The Janus-headed salvation: Sovereign and bank credit risk premia during 2008-2009. Economics Letters 110, 28-31.

Etienne, X. L., S. H. Irwin, and P. Garcia (2013). Bubbles in food commodity markets: Four decades of evidence. Journal of International Money and Finance 97, 65-87.

Fantazzini, D. (2016). The oil price crash in 2014/15: Was there a (negative) financial bubble? Energy Policy 96, 383-396.

Forbes, K. J. and R. Rigobon (2002). No contagion, only interdependence: Measuring stock market comovements. The Journal of Finance 57, 2223-2261.

Gennotte, G. and H. Leland (1990). Market liquidity, hedging, and crashes. The American Economic Review 80, 999-1021.

Gutierrez, L. (2012). Speculative bubbles in agricultural commodity markets. European Review of Agricultural Economics 40, 217-238.

Harvey, D. I., S. J. Leybourne, R. Sollis, and A. R. Taylor (2016). Tests for explosive financial bubbles in the presence of non-stationary volatility. Journal of Empirical Finance 38, 548-574. 
Harvey, D. I., S. J. Leybourne, and Y. Zu (2018). Testing explosive bubbles with time-varying volatility. Econometric Reviews, DOI: 10.1080/07474938.2018.1536099.

Harvey, D. I., S. J. Leybourne, and Y. Zu (forthcoming). Sign-based unit root tests for explosive financial bubbles in the presence of nonstationary volatility. Econometric Theory.

Hong, H. and J. C. Stein (2003). Differences of opinion, short-sales constraints, and market crashes. The Review of Financial Studies 16, 487-525.

Hu, Y. and L. Oxley (2018). Do 18th century bubbles survive the scrutiny of 21st century time series econometrics? Economics Letters 162, 131-134.

Hu, Y., L. Oxley, et al. (2016). Exuberance, bubbles or froth? Some historical results using long run house price data for Amsterdam, Norway and Paris. Working Paper in Economics 16/08, University of Waikato.

Hull, J. C. and A. D. White (2000). Valuing credit default swaps I: No counterparty default risk. The Journal of Derivatives 8, 29-40.

Manganelli, S. and G. Wolswijk (2009). What drives spreads in the euro area government bond market? Economic Policy 24, 191-240.

Meyer, G. (2013, March 25). Commodity booms, busts and bubbles. Financial Times, March 25.

Mody, A. (2009). From bear stearns to anglo irish: How eurozone sovereign spreads related to financial sector vulnerability. International Monetary Fund, Working paper 09/108.

Nolan, G. (31 May 2018). A measure of redenomination risk. Available at IHS Markit Financial Services: https://ihsmarkit.com/research-analysis/cds-redenomination.html.

Pavlidis, E., A. Yusupova, I. Paya, D. Peel, E. Martínez-García, A. Mack, and V. Grossman (2016). Episodes of exuberance in housing markets: In search of the smoking gun. The Journal of Real Estate Finance and Economics 53, 419-449.

Phillips, P. C. B. (2016). Modeling speculative bubbles with diverse investor expectations. Research in Economics 70, 375-387.

Phillips, P. C. B. and T. Magdalinos (2007). Limit theory for moderate deviations from a unit root. Journal of Econometrics 136, 115-130.

Phillips, P. C. B. and T. Magdalinos (2009). Unit root and cointegrating limit theory when initialization is in the infinite past. Econometric Theory 25, 1682-1715.

Phillips, P. C. B. and S. Shi (2017). Online supplement to the paper: Financial bubble implosion and reverse regression. Technical report.

Phillips, P. C. B. and S. Shi (2018). Financial bubble implosion and reverse regression. Econometric Theory 34, 705-753. 
Phillips, P. C. B., S. Shi, and J. Yu (2014). Specification sensitivity in right-tailed unit root testing for explosive behaviour. Oxford Bulletin of Economics and Statistics 76, 315-333.

Phillips, P. C. B., S. Shi, and J. Yu (2015a). Testing for multiple bubbles: Historical episodes of exuberance and collapse in the S\&P 500. International Economic Review 56, 1043-1078.

Phillips, P. C. B., S. Shi, and J. Yu (2015b). Testing for multiple bubbles: Limit theory of real-time detectors. International Economic Review 56, 1079-1134.

Phillips, P. C. B. and V. Solo (1992). Asymptotics for linear processes. The Annals of Statistics 20, 971-1001.

Romer, D. (1993). Rational asset-price movements without news. The American Economic Review 83, 1112-1130.

Schwarz, K. (2017). Mind the gap: Disentangling credit and liquidity in risk spreads. Working Paper, available at SSRN: https://ssrn.com/abstract=1486240.

Shi, S. (2017). Speculative bubbles or market fundamentals? An investigation of US regional housing markets. Economic Modelling 66, 101-111.

Shi, S., A. Valadkhani, R. Smyth, and F. Vahid (2016). Dating the timeline of house price bubbles in Australian capital cities. Economic Record 92, 590-605.

Wegener, C., R. Kruse, and T. Basse (2017). The walking debt crisis. Journal of Economic Behavior \&6 Organization, DOI: 10.1016/j.jebo.2017.10.008.

Yiu, M. S. and L. Jin (2013). Detecting bubbles in the Hong Kong residential property market: An explosive-pattern approach. Journal of Asian Economics 28, 115-124.

Zoli, E. and S. Sgherri (2009). Euro area sovereign risk during the crisis. IMF Working Papers Bo. 2009/222.

\section{Appendix}

This Appendix provides preliminary lemmas and main results concerning the limit behavior of recursive statistics designed to detect bubble and collapse phenomena. The generating mechanism assumed for $X_{t}=\log P_{t}$ is the $L$-process

$$
X_{t}=\left\{\begin{array}{ll}
c T^{-\eta}+X_{t-1}+\varepsilon_{t} & \text { if } t \in N \equiv\left[1, T_{c}\right] \\
X_{t-1}-L_{t}+\varepsilon_{t}, & \text { if } t \in C \equiv\left(T_{c}, T\right]
\end{array},\right.
$$

where $X_{0}=O_{p}(1), L_{t}=L b_{t}$ with $b_{t} \sim_{i . i . d} U[-\epsilon, 1]$ and $\epsilon, L>0$. The innovations $\varepsilon_{t}$ and process $L_{t}$ are assumed to be independent. In what follows let $\xi_{t}$ be the centered uniform random variate such that $\xi_{t}=b_{t}-a$ with $a=E\left(b_{t}\right)=\frac{1}{2}(1-\epsilon)$ and $\sigma_{\xi}^{2}=V\left(b_{t}\right)=\frac{1}{12}(1+\epsilon)^{2}$. 


\section{A Some Useful Lemmas}

Lemma A.1 As $T \rightarrow \infty, \frac{1}{T} \sum_{j=1}^{t=\lfloor T r\rfloor} b_{j} \rightarrow_{a . s .} r E\left(b_{j}\right)=\frac{1}{2}(1-\epsilon) r$.

Proof. By ergodicity and straightforward calculation we have as $T \rightarrow \infty$

$$
\frac{1}{T} \sum_{j=1}^{t=\lfloor T r\rfloor} b_{j}=\frac{1}{T} \sum_{j=1}^{\lfloor T r\rfloor}\left[-\epsilon+(1+\epsilon) U_{j}\right]=-\epsilon \frac{\lfloor T r\rfloor}{T}+(1+\epsilon) \frac{1}{T} \sum_{j=1}^{\lfloor T r\rfloor} U_{j} \rightarrow_{a . s .} \frac{1}{2}(1-\epsilon) r .
$$

Lemma A.2 As $T \rightarrow \infty$,

(a) $T^{-2} \sum_{j=T_{c}+2}^{T_{2}}\left(j-T_{c}-1\right) b_{j} \rightarrow \frac{1-\epsilon}{4}\left(r_{2}-r_{c}\right)^{2}$; (b) $T^{-2} \sum_{j=T_{c}+2}^{T_{2}} \sum_{i=T_{c}+1}^{j-1} b_{i} \rightarrow \frac{1-\epsilon}{4}\left(r_{2}-r_{c}\right)^{2}$; and (c) $T^{-2} \sum_{j=T_{c}+2}^{T_{2}} \sum_{i=T_{c}+1}^{j-1} b_{i} b_{j} \rightarrow \frac{(1-\epsilon)^{2}}{8}\left(r_{2}-r_{c}\right)^{2}$;

Proof. (a) Simply note that $T^{-2} \sum_{j=T_{c}+2}^{T_{2}}\left(j-T_{c}-1\right) b_{j}=a T^{-2} \sum_{j=T_{c}+2}^{T_{2}}\left(j-T_{c}-1\right)+T^{-2} \sum_{j=T_{c}+2}^{T_{2}}(j-$ $\left.T_{c}-1\right) \xi_{j}=\frac{1}{2} a\left(r_{2}-r_{c}\right)^{2}\left(1+o_{p}(1)\right)$ since $T^{-\frac{3}{2}} \sum_{j=T_{c}+2}^{T_{2}}\left(j-T_{c}-1\right) \xi_{j} \rightarrow_{d} \int_{r_{c}}^{r_{2}}\left(r-r_{c}\right) d B_{\xi}(r)$ is a weighted sum of $T_{2}-\left(T_{c}+1\right)$ iid centered uniform random variates.

(b) The result follows directly from (a) since $\sum_{j=T_{c}+2}^{T_{2}} \sum_{i=T_{c}+1}^{j-1} b_{i}=a \sum_{j=T_{c}+2}^{T_{2}} \sum_{i=T_{c}+1}^{j-1}\left(1+o_{p}(1)\right)$.

(c) In a similar fashion, $T^{-2} \sum_{j=T_{c}+2}^{T_{2}} \sum_{i=T_{c}+1}^{j-1} b_{i} b_{j}=a^{2} T^{-2} \sum_{j=T_{c}+2}^{T_{2}} \sum_{i=T_{c}+1}^{j-1}\left(1+o_{p}(1)\right) \rightarrow$ $\frac{(1-\epsilon)^{2}}{8}\left(r_{2}-r_{c}\right)^{2}$.

\section{Limit Behavior of $X_{t}$}

Lemma A.3 As $T \rightarrow \infty$,

(a) For $t \in N, X_{t=\lfloor T r\rfloor}=\sum_{s=1}^{t} \varepsilon_{s}\left\{1+o_{p}(1)\right\} \sim_{a} T^{1 / 2} B(r)$.

(b) For $t \in C, X_{t=\lfloor T r\rfloor}=-\sum_{j=T_{c}+1}^{t} L_{j}\left\{1+o_{p}(1)\right\} \sim_{a}-T L \frac{1}{2}\left(r-r_{c}\right)(1-\epsilon)$.

Proof. (a) For $t \in N, X_{t}=X_{0}+c T^{-\eta} t+\sum_{s=1}^{t} \varepsilon_{s}$, and since $T^{-1 / 2} \sum_{s=1}^{t} \varepsilon_{s} \stackrel{L}{\rightarrow} B(r)$ it follows that

$$
X_{t}=c T^{1-\eta}\left(\frac{t}{T}\right)+T^{1 / 2}\left(T^{-1 / 2} \sum_{s=1}^{t} \varepsilon_{s}\right) \sim_{a} T^{1 / 2} B(r) .
$$

(b) For $t \in C$, we have $X_{t}=\left(X_{t-1}-L_{t}\right)+\varepsilon_{t}=X_{T_{c}}-\sum_{j=T_{c}+1}^{t} L_{j}+\sum_{j=T_{c}+1}^{t} \varepsilon_{j}=-\sum_{j=T_{c}+1}^{t} L_{j}\left\{1+o_{p}(1)\right\}$ $\sim_{a}-T L \frac{1}{2}\left(r-r_{c}\right)(1-\epsilon)$, because $X_{T_{c}} \sim_{a} T^{1 / 2} B\left(r_{c}\right), \sum_{j=T_{c}+1}^{t} \varepsilon_{j} \sim_{a} T^{1 / 2}\left[B(r)-B\left(r_{c}\right)\right]$, and

$$
\sum_{j=T_{c}+1}^{t} L_{j}=T L\left(\frac{1}{T} \sum_{j=T_{c}+1}^{t} b_{j}\right) \sim_{a} T L \frac{1}{2}\left(r-r_{c}\right)(1-\epsilon)= \begin{cases}O_{p}(T) & \text { if } L \text { is a constant } \\ O_{p}(T L) & \text { if } L \rightarrow \infty\end{cases}
$$


Lemma A.4 Let $\tilde{X}_{t}=X_{t}-T_{w}^{-1} \sum_{j=T_{1}}^{T_{2}} X_{j}$. Then

(a) $\frac{1}{T_{w}} \sum_{j=T_{1}}^{T_{2}} X_{j}=\frac{1}{T_{w}} \sum_{j=T_{c}+1}^{T_{2}} X_{j}\left\{1+o_{p}(1)\right\} \sim_{a}-T L \frac{\left(r_{2}-r_{c}\right)^{2}}{r_{w}} \frac{1-\epsilon}{4}$.

(b) $\tilde{X}_{t}=X_{t}-T_{w}^{-1} \sum_{j=T_{1}}^{T_{2}} X_{j}=\left\{\begin{array}{ll}-\frac{1}{T_{w}} \sum_{j=T_{1}}^{T_{2}} X_{j}\left\{1+o_{p}(1)\right\} & \text { if } t \in N_{0} \\ {\left[X_{t}-\frac{1}{T_{w}} \sum_{j=T_{1}}^{T_{2}} X_{j}\right]\left\{1+o_{p}(1)\right\}} & \text { if } t \in C\end{array}\right.$.

Proof. (a) Write $\frac{1}{T_{w}} \sum_{j=T_{1}}^{T_{2}} X_{j}=\frac{1}{T_{w}} \sum_{j=T_{1}}^{T_{c}} X_{j}+\frac{1}{T_{w}} \sum_{j=T_{c}+1}^{T_{2}} X_{j}$. The first term is

$$
\frac{1}{T_{w}} \sum_{j=T_{1}}^{T_{c}} X_{j}=T^{1 / 2} \frac{T_{c}-T_{1}+1}{T_{w}}\left(\frac{1}{T_{c}-T_{1}+1} \sum_{j=T_{1}}^{T_{c}} \frac{X_{j}}{\sqrt{T}}\right) \sim_{a} T^{1 / 2} \frac{r_{c}-r_{1}}{r_{w}} \int_{r_{1}}^{r_{c}} B(s) d s .
$$

For the second term,

$$
\begin{aligned}
\frac{1}{T_{w}} \sum_{j=T_{c+1}}^{T_{2}} X_{j} & =-\frac{L}{T_{w}} \sum_{j=T_{c+2}}^{T_{2}} \sum_{i=T_{c}+1}^{j-1} b_{i}\left\{1+o_{p}(1)\right\}=-\frac{L}{T_{w}} T^{2}\left[T^{-2} \sum_{j=T_{c+2}}^{T_{2}} \sum_{i=T_{c}+1}^{j-1} b_{i}\right]\left\{1+o_{p}(1)\right\} \\
& \sim_{a}-L T \frac{\left(r_{2}-r_{c}\right)^{2}}{r_{w}} \frac{1-\epsilon}{4} \text { from Lemma A.2 }
\end{aligned}
$$

Thus, $\frac{1}{T_{w}} \sum_{j=T_{1}}^{T_{2}} X_{j}=\frac{1}{T_{w}} \sum_{j=T_{c}+1}^{T_{2}} X_{j}\left\{1+o_{p}(1)\right\} \sim_{a}-T L \frac{\left(r_{2}-r_{c}\right)^{2}}{r_{w}} \frac{1-\epsilon}{4}$.

(b) This follows directly from (a).

Lemma A.5 As $T \rightarrow \infty$,

(a) $\sum_{j=T_{1}}^{T_{2}} \tilde{X}_{j-1}^{2} \sim_{a} T^{3} L^{2}\left(r_{2}-r_{c}\right)^{3} \frac{(1-\epsilon)^{2}}{4}\left(\frac{1}{3}-\frac{r_{2}-r_{c}}{4 r_{w}}\right)$,

(b) $\sum_{j=T_{1}}^{T_{2}} \tilde{X}_{j-1} \varepsilon_{j} \sim_{a}-T^{3 / 2} L \frac{1}{2}(1-\epsilon)\left\{\left(r_{2}-r_{c}\right) B\left(r_{2}\right)-\int_{r_{c}}^{r_{2}} B(s) d s-\frac{\left(r_{2}-r_{c}\right)^{2}}{2 r_{w}}\left[B\left(r_{2}\right)-B\left(r_{1}\right)\right]\right\}$,

(c) $\sum_{j=T_{1}}^{T_{2}} \tilde{X}_{j-1}\left(\tilde{X}_{j}-\tilde{X}_{j-1}\right) \sim_{a} T^{2} L^{2} \frac{\left(r_{2}-r_{c}\right)^{2}(1-\epsilon)^{2}}{8 r_{w}}\left(r_{c}-r_{1}\right)$.

Proof. (a) Write $\sum_{j=T_{1}}^{T_{2}} \tilde{X}_{j-1}^{2}=\sum_{j=T_{1}}^{T_{c}+1} \tilde{X}_{j-1}^{2}+\sum_{j=T_{c}+2}^{T_{2}} \tilde{X}_{j-1}^{2}$. The first term is

$$
\sum_{j=T_{1}}^{T_{c}+1} \tilde{X}_{j-1}^{2}=\sum_{j=T_{1}}^{T_{c}+1}\left[-\frac{1}{T_{w}} \sum_{j=T_{1}}^{T_{2}} X_{j}\right]^{2}\left\{1+o_{p}(1)\right\} \sim_{a} T^{3} L^{2}\left(r_{c}-r_{1}\right) \frac{\left(r_{2}-r_{c}\right)^{4}(1-\epsilon)^{2}}{16 r_{w}^{2}} .
$$

The second term is

$$
\begin{aligned}
& \sum_{j=T_{c}+2}^{T_{2}} \tilde{X}_{j-1}^{2}=\sum_{j=T_{c}+2}^{T_{2}}\left[X_{j-1}-\frac{1}{T_{w}} \sum_{i=T_{1}}^{T_{2}} X_{i}\right]^{2}\left\{1+o_{p}(1)\right\} \\
& =\left[\sum_{j=T_{c}+2}^{T_{2}} X_{j-1}^{2}-2\left(\frac{1}{T_{w}} \sum_{i=T_{1}}^{T_{2}} X_{i}\right)\left(\sum_{j=T_{c}+2}^{T_{2}} X_{j-1}\right)+\sum_{j=T_{c}+2}^{T_{2}}\left(\frac{1}{T_{w}} \sum_{i=T_{1}}^{T_{2}} X_{i}\right)^{2}\right]\left\{1+o_{p}(1)\right\}
\end{aligned}
$$




$$
\sim_{a} T^{3} L^{2} \frac{\left(r_{2}-r_{c}\right)^{3}}{4}(1-\epsilon)^{2}\left[\frac{1}{3}-\frac{r_{2}-r_{c}}{2 r_{w}}+\frac{\left(r_{2}-r_{c}\right)^{2}}{4 r_{w}^{2}}\right],
$$

since

$$
\begin{aligned}
& \sum_{j=T_{c}+2}^{T_{2}} X_{j-1}^{2}=\sum_{j=T_{c}+2}^{T_{2}}\left(-\sum_{i=T_{c}+1}^{j-1} L_{i}\right)^{2}\left\{1+o_{p}(1)\right\} \\
= & \sum_{j=T_{c}+2}^{T_{2}}\left(j-1-T_{c}\right)^{2}\left(\frac{1}{j-1-T_{c}} \sum_{i=T_{c}+1}^{j-1} L_{i}\right)^{2}\left\{1+o_{p}(1)\right\} \\
= & L^{2}\left(\frac{1}{j-1-T_{c}} \sum_{i=T_{c}+1}^{j-1} b_{i}\right)^{2} \sum_{j=T_{c}+2}^{T_{2}}\left(j-1-T_{c}\right)^{2}\left\{1+o_{p}(1)\right\} \sim_{a} T^{3} L^{2} \frac{\left(r_{2}-r_{c}\right)^{3}}{12}(1-\epsilon)^{2} \\
& \sum_{j=T_{c}+2}^{T_{2}} X_{j-1}=-\sum_{j=T_{c}+2}^{T_{2}} \sum_{i=T_{c}+1}^{j-1} L_{i}\left\{1+o_{p}(1)\right\}=-L\left[\sum_{j=T_{c+2}}^{T_{2}} \sum_{i=T_{c}+1}^{j-1} b_{i}\right]\left\{1+o_{p}(1)\right\} \\
= & -L T^{2}\left[T^{-2} \sum_{j=T_{c+2}}^{T_{2}} \sum_{i=T_{c}+1}^{j-1} b_{i}\right]\left\{1+o_{p}(1)\right\} \sim_{a}-T^{2} L\left(r_{2}-r_{c}\right)^{2} \frac{1-\epsilon}{4} \\
-2 & \left.\frac{1}{T_{w}} \sum_{i=T_{1}}^{T_{2}} X_{i}\right)\left(\sum_{j=T_{c}+2}^{T_{2}} X_{j-1}\right) \sim_{a}-T^{3} L^{2} \frac{\left(r_{2}-r_{c}\right)^{4}(1-\epsilon)^{2}}{8 r_{w}}
\end{aligned}
$$

and

$$
\sum_{j=T_{c}+2}^{T_{2}}\left(\frac{1}{T_{w}} \sum_{i=T_{1}}^{T_{2}} X_{i}\right)^{2}=\left(T_{2}-T_{c}-1\right)\left(\frac{1}{T_{w}} \sum_{i=T_{1}}^{T_{2}} X_{i}\right)^{2} \sim_{a} T^{3} L^{2} \frac{\left(r_{2}-r_{c}\right)^{5}(1-\epsilon)^{2}}{16 r_{w}^{2}} .
$$

Therefore,

$$
\sum_{j=T_{1}}^{T_{2}} \tilde{X}_{j-1}^{2} \sim_{a} T^{3} L^{2}\left(r_{2}-r_{c}\right)^{3} \frac{(1-\epsilon)^{2}}{4}\left(\frac{1}{3}-\frac{r_{2}-r_{c}}{4 r_{w}}\right) .
$$

(b) Write $\sum_{j=T_{1}}^{T_{2}} \tilde{X}_{j-1} \varepsilon_{j}=\sum_{j=T_{1}}^{T_{c}+1} \tilde{X}_{j-1} \varepsilon_{j}+\sum_{j=T_{c}+2}^{T_{2}} \tilde{X}_{j-1} \varepsilon_{j}$. The first term is

$$
\begin{aligned}
& \sum_{j=T_{1}}^{T_{c}+1} \tilde{X}_{j-1} \varepsilon_{j}=\sum_{j=T_{1}}^{T_{c}+1}\left[-\frac{1}{T_{w}} \sum_{i=T_{1}}^{T_{2}} X_{i}\right] \varepsilon_{j}\left\{1+o_{p}(1)\right\}=\left(-\frac{1}{T_{w}} \sum_{i=T_{1}}^{T_{2}} X_{i}\right) \sum_{j=T_{1}}^{T_{c}+1} \varepsilon_{j}\left\{1+o_{p}(1)\right\} \\
& \sim_{a} T^{3 / 2} L \frac{\left(r_{2}-r_{c}\right)^{2}(1-\epsilon)}{4 r_{w}}\left[B\left(r_{c}\right)-B\left(r_{1}\right)\right],
\end{aligned}
$$

since $\frac{1}{T_{w}} \sum_{i=T_{1}}^{T_{2}} X_{i} \sim_{a}-T L \frac{\left(r_{2}-r_{c}\right)^{2}(1-\epsilon)}{4 r_{w}}$, and $\sum_{j=T_{1}}^{T_{c}+1} \varepsilon_{j} \sim_{a} T^{1 / 2}\left[B\left(r_{c}\right)-B\left(r_{1}\right)\right]$. The second term is

$$
\sum_{j=T_{c}+2}^{T_{2}} \tilde{X}_{j-1} \varepsilon_{j}=\sum_{j=T_{c}+2}^{T_{2}}\left(X_{j-1}-\frac{1}{T_{w}} \sum_{i=T_{1}}^{T_{2}} X_{i}\right) \varepsilon_{j}=\sum_{j=T_{c}+2}^{T_{2}} X_{j-1} \varepsilon_{j}-T^{1 / 2}\left(\frac{1}{T_{w}} \sum_{i=T_{1}}^{T_{2}} X_{i}\right)\left(T^{-1 / 2} \sum_{j=T_{c}+2}^{T_{2}} \varepsilon_{j}\right)
$$


$\sim_{a}-T^{3 / 2} L \frac{1-\epsilon}{2}\left\{\left(r_{2}-r_{c}\right) B\left(r_{2}\right)-\int_{r_{c}}^{r_{2}} B(s) d s-\frac{\left(r_{2}-r_{c}\right)^{2}}{2 r_{w}}\left[B\left(r_{2}\right)-B\left(r_{c}\right)\right]\right\}$

because

$$
\begin{gathered}
\sum_{j=T_{c+2}}^{T_{2}} X_{j-1} \varepsilon_{j}=\sum_{j=T_{c+2}}^{T_{2}}\left[-\sum_{i=T_{c}+1}^{j-1} L_{i}\left\{1+o_{p}(1)\right\}\right] \varepsilon_{j} \\
=-L \sum_{j=T_{c}+2}^{T_{2}}\left(j-1-T_{c}\right) \varepsilon_{j}\left(\frac{1}{j-1-T_{c}} \sum_{i=T_{c}+1}^{j-1} b_{i}\right)\left\{1+o_{p}(1)\right\} \\
=-T^{3 / 2} L \frac{1-\epsilon}{2}\left[T^{-3 / 2} \sum_{j=T_{c}}^{T_{2}}\left(j-1-T_{c}\right) \varepsilon_{j}\right]\left\{1+o_{p}(1)\right\} \\
\sim_{a}-T^{3 / 2} L \frac{1-\epsilon}{2}\left[\left(r_{2}-r_{c}\right) B\left(r_{2}\right)-\int_{r_{c}}^{r_{2}} B(s) d s\right]
\end{gathered}
$$

and

$$
\begin{aligned}
-T^{1 / 2}\left(\frac{1}{T_{w}} \sum_{i=T_{1}}^{T_{2}} X_{i}\right)\left(T^{-1 / 2} \sum_{j=T_{c}}^{T_{2}} \varepsilon_{j}\right)= & -T^{1 / 2}\left(\frac{1}{T_{w}} \sum_{i=T_{1}}^{T_{2}} X_{i}\right)\left(T^{-1 / 2} \sum_{j=T_{c}}^{T_{2}} \varepsilon_{j}\right) \\
& \sim_{a} T^{3 / 2} L \frac{\left(r_{2}-r_{c}\right)^{2}(1-\epsilon)}{4 r_{w}}\left[B\left(r_{2}\right)-B\left(r_{c}\right)\right] .
\end{aligned}
$$

Thus,

$$
\sum_{j=T_{1}}^{T_{2}} \tilde{X}_{j-1} \varepsilon_{j} \sim_{a}-T^{3 / 2} L \frac{1}{2}(1-\epsilon)\left\{\left(r_{2}-r_{c}\right) B\left(r_{2}\right)-\int_{r_{c}}^{r_{2}} B(s) d s-\frac{\left(r_{2}-r_{c}\right)^{2}}{2 r_{w}}\left[B\left(r_{2}\right)-B\left(r_{1}\right)\right]\right\} .
$$

(c) Write

$$
\begin{aligned}
\sum_{j=T_{1}}^{T_{2}} \tilde{X}_{j-1}\left(\tilde{X}_{j}-\tilde{X}_{j-1}\right) & =\sum_{j=T_{1}}^{T_{c}+1} \tilde{X}_{j-1} \varepsilon_{j}+\sum_{j=T_{c}+2}^{T_{2}} \tilde{X}_{j-1}\left(-L_{j}+\varepsilon_{j}\right) \\
& =\sum_{j=T_{1}}^{T_{2}} \tilde{X}_{j-1} \varepsilon_{j}-\sum_{j=T_{c}+2}^{T_{2}} \tilde{X}_{j-1} L_{j} .
\end{aligned}
$$

The first term is

$$
\sum_{j=T_{1}}^{T_{2}} \tilde{X}_{j-1} \varepsilon_{j} \sim_{a}-T^{3 / 2} L \frac{1}{2}(1-\epsilon)\left\{\left(r_{2}-r_{c}\right) B\left(r_{2}\right)-\int_{r_{c}}^{r_{2}} B(s) d s-\frac{\left(r_{2}-r_{c}\right)^{2}}{2 r_{w}}\left[B\left(r_{2}\right)-B\left(r_{1}\right)\right]\right\} .
$$

The second term is

$$
\begin{aligned}
\sum_{j=T_{c}+2}^{T_{2}} \tilde{X}_{j-1} L_{j} & =\sum_{j=T_{c}+2}^{T_{2}}\left[X_{j-1}-\frac{1}{T_{w}} \sum_{i=T_{1}}^{T_{2}} X_{i}\right] L_{j}\left\{1+o_{p}(1)\right\} \\
& =\left[\sum_{j=T_{c}+2}^{T_{2}} X_{j-1} L_{j}-\left(\frac{1}{T_{w}} \sum_{i=T_{1}}^{T_{2}} X_{i}\right) \sum_{j=T_{c}+2}^{T_{2}} L_{j}\right]\left\{1+o_{p}(1)\right\}
\end{aligned}
$$




$$
\sim^{a}-T^{2} L^{2} \frac{\left(r_{2}-r_{c}\right)^{2}(1-\epsilon)^{2}}{8 r_{w}}\left(r_{c}-r_{1}\right)
$$

because

$$
\sum_{j=T_{c}+2}^{T_{2}} X_{j-1} L_{j}=\sum_{j=T_{c}+2}^{T_{2}}\left(-\sum_{i=T_{c}+1}^{j-1} L_{i}\right) L_{j}\left\{1+o_{p}(1)\right\} \sim_{a}-T^{2} L^{2} \frac{\left(r_{2}-r_{c}\right)^{2}(1-\epsilon)^{2}}{8} \text { from Lemma A.2, }
$$

and

$$
\begin{aligned}
-\left(\frac{1}{T_{w}} \sum_{i=T_{1}}^{T_{2}} X_{i}\right)\left(\sum_{j=T_{c}+2}^{T_{2}} \tilde{L}_{j}\right) & =-\left(T_{2}-T_{c}\right)\left(\frac{1}{T_{w}} \sum_{i=T_{1}}^{T_{2}} X_{i}\right)\left(\frac{1}{T_{2}-T_{c}} \sum_{j=T_{c}+2}^{T_{2}} L_{j}\right) \\
& \sim_{a} T^{2} L^{2} \frac{\left(r_{2}-r_{c}\right)^{3}(1-\epsilon)^{2}}{8 r_{w}} .
\end{aligned}
$$

Thus,

$$
\sum_{j=T_{1}}^{T_{2}} \tilde{X}_{j-1}\left(\tilde{X}_{j}-\tilde{X}_{j-1}\right) \sim_{a} T^{2} L^{2} \frac{\left(r_{2}-r_{c}\right)^{2}(1-\epsilon)^{2}}{8 r_{w}}\left(r_{c}-r_{1}\right)
$$

\section{B Test asymptotics}

The fitted regression for the recursive unit root tests has the following form (see PSY, 2015a \& 2015b for details)

$$
\tilde{X}_{t}=\hat{\mu}_{r_{1}, r_{2}}+\hat{\rho}_{r_{1}, r_{2}} \tilde{X}_{t-1}+\hat{\varepsilon}_{t},
$$

where $\tilde{X}_{t}$ is demeaned $X_{t}$, and the intercept $\hat{\mu}_{r_{1}, r_{2}}$ and slope coefficient $\hat{\rho}_{r_{1}, r_{2}}$ are obtained using data over the subperiod $\left[r_{1}, r_{2}\right]$.

Remark 1 Based on Lemma A.5, the limit behavior of $\hat{\rho}_{r_{1}, r_{2}}-1$ is obtained using

$$
\begin{aligned}
\hat{\rho}_{r_{1}, r_{2}}-1 & =\frac{\sum_{j=T_{1}}^{T_{2}} \tilde{X}_{j-1}\left(\tilde{X}_{j}-\tilde{X}_{j-1}\right)}{\sum_{j=T_{1}}^{T_{2}} \tilde{X}_{j-1}^{2}} \\
& = \begin{cases}\frac{O_{p}\left(T^{2}\right)}{O_{p}\left(T^{3}\right)}=O_{p}\left(T^{-1}\right) & \text { if } L \text { is a constant } \\
\frac{O_{p}\left(T^{2} L^{2}\right)}{O_{p}\left(T^{3} L^{2}\right)}=O_{p}\left(T^{-1}\right) & \text { if } L \rightarrow \infty\end{cases}
\end{aligned}
$$

The sign of $\hat{\rho}_{r_{1}, r_{2}}-1$ is identical to that of the quantity $\sum_{j=T_{1}}^{T_{2}} X_{j-1}\left(X_{j}-X_{j-1}\right)$, which is positive.

Remark 2 The residual error variance of the regression over $\left[T_{1}, T_{2}\right]$ is

$$
\hat{\sigma}_{r_{1}, r_{2}}^{2}=T_{w}^{-1} \sum_{j=T_{1}}^{T_{2}}\left(\tilde{X}_{j}-\hat{\rho}_{r_{1}, r_{2}} \tilde{X}_{j-1}\right)^{2}
$$




$$
\begin{aligned}
& =T_{w}^{-1}\left[\sum_{j=T_{1}}^{T_{c}-1}\left[\varepsilon_{j}-\left(\hat{\rho}_{r_{1}, r_{2}}-1\right) \tilde{X}_{j-1}\right]^{2}+\sum_{j=T_{c}}^{T_{2}}\left[\varepsilon_{j}-\left(\hat{\rho}_{r_{1}, r_{2}}-1\right) \tilde{X}_{j-1}-L_{j}\right]^{2}\right] \\
& =T_{w}^{-1} \sum_{j=T_{1}}^{T_{2}} \varepsilon_{j}^{2}+\left(\hat{\rho}_{r_{1}, r_{2}}-1\right)^{2} T_{w}^{-1} \sum_{j=T_{1}}^{T_{2}} \tilde{X}_{j-1}^{2}-2 T_{w}^{-1} \sum_{j=T_{c}}^{T_{2}} \varepsilon_{j} L_{j} \\
& -2\left(\hat{\rho}_{r_{1}, r_{2}}-1\right) T_{w}^{-1} \sum_{j=T_{1}}^{T_{2}} \tilde{X}_{j-1} \varepsilon_{j}+2\left(\hat{\rho}_{r_{1}, r_{2}}-1\right) T_{w}^{-1} \sum_{j=T_{c}}^{T_{2}} \tilde{X}_{j-1} E_{j} \\
& =\left\{\begin{array}{ll}
\left(\hat{\rho}_{r_{1}, r_{2}}-1\right)^{2} T_{w}^{-1} \sum_{j=T_{1}}^{T_{2}} \tilde{X}_{j-1}^{2}-2\left(\hat{\rho}_{r_{1}, r_{2}}-1\right) T_{w}^{-1} \sum_{j=T_{1}}^{T_{2}} \tilde{X}_{j-1} \varepsilon_{j}
\end{array}\right\}\left\{1+o_{p}(1)\right\} \\
& = \begin{cases}O_{p}(1) & \text { if } L \text { is a constant } \\
O_{p}\left(L^{2}\right) & \text { if } L \rightarrow \infty\end{cases}
\end{aligned}
$$

The above orders follow because: (i) when $L$ is a constant

$$
\begin{gathered}
\left(\hat{\rho}_{r_{1}, r_{2}}-1\right)^{2} T_{w}^{-1} \sum_{j=T_{1}}^{T_{2}} \tilde{X}_{j-1}^{2}=O_{p}\left(T^{-2}\right) O_{p}\left(T^{2}\right)=O_{p}(1), \\
2\left(\hat{\rho}_{r_{1}, r_{2}}-1\right) T_{w}^{-1} \sum_{j=T_{1}}^{T_{2}} \tilde{X}_{j-1} \varepsilon_{j}=O_{p}\left(T^{-1}\right) O_{p}\left(T^{1 / 2}\right)=O_{p}\left(T^{-1 / 2}\right), \\
2\left(\hat{\rho}_{r_{1}, r_{2}}-1\right) T_{w}^{-1} \sum_{j=T_{c}}^{T_{2}} \tilde{X}_{j-1} L_{j}=O_{p}\left(T^{-1}\right) O_{p}(T)=O_{p}(1) ;
\end{gathered}
$$

and (ii) when $L \rightarrow \infty$

$$
\begin{gathered}
\left(\hat{\rho}_{r_{1}, r_{2}}-1\right)^{2} T_{w}^{-1} \sum_{j=T_{1}}^{T_{2}} \tilde{X}_{j-1}^{2}=O_{p}\left(T^{-2}\right) O_{p}\left(T^{2} L^{2}\right)=O_{p}\left(L^{2}\right), \\
2\left(\hat{\rho}_{r_{1}, r_{2}}-1\right) T_{w}^{-1} \sum_{j=T_{1}}^{T_{2}} \tilde{X}_{j-1} \varepsilon_{j}=O_{p}\left(T^{-1}\right) O_{p}\left(T^{1 / 2} L\right)=O_{p}\left(T^{-1 / 2} L\right), \\
2\left(\hat{\rho}_{r_{1}, r_{2}}-1\right) T_{w}^{-1} \sum_{j=T_{c}}^{T_{2}} \tilde{X}_{j-1} L_{j}=O_{p}\left(T^{-1}\right) O_{p}\left(T L^{2}\right)=O_{p}\left(L^{2}\right) .
\end{gathered}
$$

Then, the limit distribution of the unit root $\mathrm{t}$ statistic is

$$
D F_{r_{1}}^{r_{2}}=\left(\frac{\sum_{j=T_{1}}^{T_{2}} \tilde{X}_{j-1}^{2}}{\hat{\sigma}_{r_{1} r_{2}}^{2}}\right)^{1 / 2}\left(\hat{\rho}_{r_{1}, r_{2}}-1\right)=O_{p}\left(T^{1 / 2}\right) \rightarrow+\infty .
$$

which follows directly from the results in Lemma A.5 and Remark 1 and 2. This proves Theorem 1 . 